\title{
Large structure in the far wakes of two-dimensional bluff bodies
}

\author{
By JOHN M. CIMBALA $\dagger$, HASSAN M. NAGIB \\ AND ANATOL ROSHKO§ \\ + Mechanical Engineering Department, Pennsylvania State University, \\ University Park, PA 16802 , USA \\ ‡ Mechanical and Aerospace Engineering, Illinois Institute of Technology, \\ Chicago, IL 60616, USA \\ $\S$ Graduate Aeronautical Labs, California Institute of Technology, Pasadena, CA 91125, USA
}

(Received 19 April 1985 and in revised form 28 September 1987)

Smoke-wire flow visualization and hot-wire anemometry have been used to study near and far wakes of two-dimensional bluff bodies. For the case of a circular cylinder at $70<R e<2000$, a very rapid (exponential) decay of velocity fluctuations at the Kármán-vortex-street frequency is observed. Beyond this region of decay, largerscale (lower wavenumber) structure can be seen. In the far wake (beyond one hundred diameters) a broad band of frequencies is selectively amplified and then damped, the centre of the band shifting to lower frequencies as downstream distance is increased.

The far-wake structure does not depend directly on the scale or frequency of Kármán vortices shed from the cylinder; i.e. it does not result from amalgamation of shed vortices. The growth of this structure is due to hydrodynamic instability of the developing mean wake profile. Under certain conditions amalgamation can take place, but is purely incidental, and is not the driving mechanism responsible for the growth of larger-scale structure. Similar large structure is observed downstream of porous flat plates ( $R e \approx 6000$ ), which do not initially shed Kármán-type vortices into the wake.

Measured prominent frequencies in the far cylinder wake are in good agreement with those estimated by two-dimensional locally parallel inviscid linear stability theory, when streamwise growth of wake width is taken into account. Finally, threedimensionality in the far wake of a circular cylinder is briefly discussed and a mechanism for its development is suggested based on a secondary parametric instability of the subharmonic type.

\section{Introduction}

\subsection{Historical overview}

Wakes generated by two-dimensional bluff bodies have been the focus of hundreds of experiments since the early 1900s. For a detailed review or bibliography of work on this subject, the reader is referred to Morkovin (1964) or Berger \& Wille (1972). The majority of experimental efforts in the past have dealt primarily with near wakes (only a few diameters downstream). A more limited amount of work has been done on the far wakes of bluff bodies (up to several hundred diameters), and that is the subject on which we wish to focus here.

Let us consider the two-dimensional problem. It is well known from dimensional 
analysis and simple scaling laws that a plane far wake grows as $x^{\frac{1}{2}}$ (see for example Cantwell 1979). We would expect therefore, as we go downstream in the wake, that the size of the largest-scale structure should increase, while its passage frequency should decrease, the convection velocity being approximately constant. This in fact has been experimentally verified. Taneda (1959), using flow visualization in the wakes of two-dimensional cylinders and flat plates, observed a far-wake structure which appeared to be much like the original Kármán vortex street, but of larger scale. He viewed the process as one of changing hydrodynamic stability, i.e. the original street decays (breaks down) and the wake 'rearranges' itself into a new configuration appropriate to the new (downstream) position. Taneda found that for laminar wakes $(50<R e<150)$ the ratio of the secondary-vortex-street scale to that of the primary (Kármán) street $\left(a_{2} / a_{1}\right)$ varied from 1.5 to 3.5 ; for turbulent wakes this ratio was roughly 10 , and the secondary street did not appear until much farther downstream, after the wake had had time to grow significantly. He also reported that this sequence sometimes repeated itself.

Other investigators have also reported large-scale organized structure in the far wakes of bluff bodies. Grant (1958) observed quasi-periodic large structures several hundred diameters downstream of his cylinder at $R e=1300$, which he described as 'jets of turbulent fluid proceeding outward from the central plane of the wake'. Successive 'jets' arise and decay, their overall size becoming larger with increasing downstream distance.

Gerrard (1966) studied the downstream development of a cylinder wake at $R e=113$. At $x / d=\mathbf{4 5 0}$ he found that the fundamental (Kármán) frequency was lost in the background noise, but a lower-frequency fluctuation was found. The possibility of a secondary vortex street was suggested, the ratio of secondary to primary frequencies being about 0.033 .

Zdravkovich (1968) studied the development of the wake behind a set of three circular cylinders in close proximity. He found that the shed vortices rapidly decayed as they interacted with each other. Subsequently, a new vortex street of larger scale appeared downstream. Zdravkovich explained the formation of the secondary vortex street as a coupling of two mechanisms - shear-layer instability and a rolling-up process induced by the distributed vorticity in the wake. Similar experiments were done more recently by Williamson (1985).

An obvious extension of Zdravkovich's work is to the wake of a cluster of many cylinders or, in the general case, the wake of a porous two-dimensional body, such as a screen or perforated flat plate aligned normal to the flow. Investigations of the latter have been reported by Castro (1971) and more recently by Valensi (1974). Castro categorized two distinct regimes of flow based on the solidity of the plate: $(a)$ for high solidity (low porosity) a Kármán vortex street dominates the near wake; $(b)$ for low solidity $(\sigma<0.8)$ the plate does not shed Kármán vortices, but there is a dominant frequency present, which he attributed to far-wake instability. Valensi examined the latter regime in more detail with a $53 \%$ solid plate; he shows power spectra and smoke pictures. The dominant near-wake frequency corresponds to instabilities in the shear layers on either side of the wake; further downstream (beyond five plate widths), the dominant frequency is associated with a vortex street which forms after the shear layers have merged. Smoke visualization confirms his hot-wire results.

Another interesting set of experiments was reported by Durgin \& Karlsson (1971). They subjected the cylinder wake to a deceleration, thereby distorting the Kármán vortex street. Their results show an annihilation of the concentrated vortices, and 
the subsequent creation of a new vortex street of lower frequency and longer wavelength. They, like Taneda, attributed this effect to changing hydrodynamic stability. Although the non-decelerated wake of 'Taneda does not experience bending and stretching of vortex filaments, as does the decelerated wake, the similarity of the two results is remarkable.

In a closely related experiment, Keffer (1965) used a constant-area distorting duct to impose a strain field upon the wake. His flow-visualization results show a secondary vortex-street-like structure emerging with a frequency 0.36 times the Kármán frequency at $R e=350$.

Interest in Taneda's discovery has resurfaced in the past decade. Based on an inviscid model, Weihs (1973) suggested that multiple Kármán-vortex-street modes are possible. In an attempt to compare his model with experiment, he plotted Taneda's results and obtained the fit (for $60<R e<120$ )

$$
\frac{a_{2}}{a_{1}}=\frac{220}{R e}
$$

although there is considerable scatter in the data. Matsui \& Okude (1980) made careful hot-wire measurements, at Reynolds numbers between 100 and 160, of the velocity fluctuations associated with this secondary-street phenomenon. They also reported an inverse Reynolds-number relation, but their constant was very different from Taneda's (equation 1.1):

$$
\frac{a_{2}}{a_{1}}=\frac{295}{R e} \text {. }
$$

Thus it seems that the development of the secondary vortex street is not independent of experimental facility. (Taneda's cylinders were towed in a water channel, while Matsui \& Okude used a low-speed wind tunnel.)

Meanwhile Townsend (1979), using multiple hot-wire arrays, found periodic flow patterns resembling Kármán vortex streets for a much higher Reynolds number $(R e=8000$ at $x / d=170)$. 'Groups' of three to five vortical structures were observed, the passage frequency within a group being uniform but varying considerably from one group to the next. Regions of random fluctuations seem to fill the gaps between successive groups. Long-time-averaged spectra therefore do not show a peak at any particular frequency. Townsend estimated that these eddy groups contribute 15-20\% of the total turbulent energy. Such grouping of structures had previously been suggested by Gupta, Laufer \& Kaplan (1971) for the turbulent boundary layer.

Matsui \& Okude (1981) later abandoned Taneda's original conjecture that the secondary street results from hydrodynamic instability, following the 'breakdown' of the primary street. In its stead they proposed that pairing is the mechanism for the change in scale. Matsui \& Okude's conclusion, however, was based partly on flow visualization, where the flow tracer was introduced upstream of the cylinder. As will be pointed out in $\S 3$, this type of visualization can be very misleading. Furthermore, with a pairing mechanism the frequency of the secondary street ought to be half that of the primary street, but this is not the case for all Reynolds numbers. Matsui \& Okude (1980) measured the frequencies of the primary and secondary streets. From their figure 10 one can calculate the ratio of these two frequencies; $f_{2} / f_{1}$ ranges from 0.2 to 0.52 .

At about the same time, Cimbala, Nagib \& Roshko (1981) reported strong experimental evidence in support of Taneda's stability hypothesis. Namely, they employed the smoke-wire flow-visualization technique of Corke et al. (1977) and hot- 
wire anemometry to show that concentrated vortices of the Kármán vortex street decay exponentionally. As will be discussed in $\$ 3$, the very rapid decay of the Kármán vortex street has been known for quite some time (see for example Roshko 1953); this fact has tended to be obscured, however, owing to misinterpretation of streakline flow visualization. Cimbala et al. showed that by 100-150 diameters downstream of a cylinder at $R e \approx 100$ the initial vortex street can no longer be found. Thus they concluded that the development of any subsequent structure can not be directly dependent upon the original vortex street (i.e. amalgamation) but rather must be the result of a local wake instability of the developing mean velocity profile. Cimbala (1984) made hot-wire surveys of the developing wake; comparison of linear stability calculations with measured prominent frequencies showed good agreement, which provided further support for this statement. Details of these experiments are reported in the present paper.

Matsui \& Okude (1983) again studied the wake at $R e=140$. Using simultaneous hot-wire anemometry and smoke visualization at $x / d=75$, they showed that the smoke lumps are indeed vortices at this location; therefore they concluded, based on flow visualization of these lumps, that pairing is the predominant mechanism for the change of scale. The frequency ratio $f_{2} / f_{1}$ is not always exactly one-half because not all of the vortices pair up; a few are left stranded, causing some irregularity. However, when Matsui \& Okude acoustically excited the wake at $\frac{1}{2}$ or $\frac{1}{3}$ of the Kármán frequency, they observed regular pairing or tripling, respectively.

In contrast to the conclusions of Matsui \& Okude, Nagib \& Desruelle (1982) and later Desruelle (1983) confirmed the findings of Cimbala et al. (1981) in a different flow facility. In addition they used acoustic excitation to experimentally derive the stability characteristics of the wake. Namely, amplification rates are plotted as a function of disturbance frequency and of downstream location. As expected, the band of amplified frequencies shifts to lower values and broadens as downstream distance is increased. Also, at about the same time, Champagne, Marasli \& Wygnanski (1982) and Wygnanski, Champagne \& Marasli (1986) introduced disturbances at the trailing edge of a flat plate, and investigated spatial growth rate. They found that the turbulent-wake response corresponds surprisingly well to that calculated from linear stability theory applied to the mean flow and with its growth taken into account (Gaster, Kit \& Wygnanski 1985).

Several theoretical and numerical investigations of this matter have also been made; for example, see Weihs (1973), Aref \& Siggia (1981), Schatzman (1981), Saffman \& Schatzman (1982), and most recently Meiburg (1987). For the most part these models are inviscid, two-dimensional, and assume an infinite array of vortices in temporal, rather than spatial development; only the subharmonic instability has been given much attention, although Saffman \& Schatzman discuss other possibilities. Robinson \& Saffman (1982) investigated the three-dimensional stability of an initially two-dimensional row of staggered vortices.

\subsection{Objective}

For the case of a plane free shear layer, amalgamation of small vortices into larger ones appears to be an important feature of the growth of coherent structures (see for example Winant \& Browand 1974). Such amalgamation occurs most frequently when the shear layer is locally receptive to scales close to the subharmonic of existing scales in the flow, i.e. subharmonic resonance. It is then a matter of semantics as to which mechanism is prominent - amalgamation or hydrodynamic instability. But to what extent amalgamation influences the scale of structure in a wake is of concern 
here. As discussed above, there is some disagreement among researchers as to the mechanism(s) responsible for the large-scale structure observed in far wakes of twodimensional bluff bodies. The two views are summarized here:

(a) Hydrodynamic instability of the wake profile is responsible for the growth of downstream structure, independent of and not directly resulting from amalgamation of vortices shed from the body (Kármán vortex street). Amalgamation, if it occurs, is incidental and is not itself the primary mechanism for the change of scale.

(b) The large structure in the far wake is a result of amalgamation of Kármán vortices into larger vortical structures.

Our overall objective, then, was to describe definitively the mechanism that causes the change of scale. The experimental tools we have employed toward this end are hot-wire anemometry and smoke-wire visualization. We are primarily concerned with the two-dimensional aspect of the problem; three-dimensionality is discussed briefly.

\section{Experimental facility and instrumentation}

The flow-visualization experiments, as well as the hot-wire measurements, were conducted in an open-return, low-turbulence wind tunnel at the Guggenheim Aeronautical Laboratories of Caltech. The test section is $0.5 \mathrm{~m}$ square and $2 \mathrm{~m}$ long; flow velocity is variable from $0.5 \mathrm{~m} / \mathrm{s}$ to $12 \mathrm{~m} / \mathrm{s}$, and background turbulence intensity $u_{\mathrm{rms}}^{\prime} / U_{\infty}$ of the free stream is $0.1-0.2 \%$ at $U_{\infty}=2 \mathrm{~m} / \mathrm{s}$.

The circular cylinders used in the experiments varied in diameter from $0.06 \mathrm{~cm}$ to $0.5 \mathrm{~cm}$, giving a Reynolds-number range from 70 to over 2000 (based on cylinder diameter, $\left.R e=U_{\infty} d / v\right)$. The cylinders, made of drill rod, spanned the test section and passed through the walls at either end, where they were held in tension by fasteners outside the tunnel walls. Drill rod provided the required strength, uniformity of diameter, and smoothness. Before each run, the cylinder was polished carefully to remove any dust build-up or corrosion.

In addition to circular cylinders, porous flat plates were also stretched across the test section, with their broad sides facing the flow. The plates were approximately $2 \mathrm{~cm}$ in width and were cut from screens. (Reynolds number based on this width ranged from 3000 to 9000 .) We looked at a number of porous plates of various solidities and mesh sizes, from which we picked one representative case for our smoke-visualization experiments and hot-wire measurements, namely a $47 \%$ solidity screen with wire diameter $0.43 \mathrm{~mm}$ and mesh size $1.59 \mathrm{~mm}$. The screen was held in tension by fasteners passing through the tunnel walls.

A simple technique for introducing closely spaced streaklines in wind tunnels is called 'smoke-wire flow visualization,' and is described by Corke et al. (1977). In the present experiments, the smoke wire used for flow visualization was a $0.13 \mathrm{~mm}$ diameter stainless-steel wire which stretched from top to bottom of the test section through the tunnel walls. A strobe and camera were synchronized to the operation of the wire, allowing instantaneous snap-shots of smoke streaklines to be recorded. More details of our set-up can be found in Cimbala (1984).

Free-stream velocities were measured with a United Sensors Pitot-static tube and an electronic Barocel manometer. Fluctuating velocities were measured with miniature single- and dual-sensor hot-wire probes (T.S.I. models 1260-T1.5 and 1248T1.5, respectively). The single-sensor probe was a $4 \mu \mathrm{m}$ platinum-plated tungsten wire, with a length of $1.5 \mathrm{~mm}(L / d=375)$. It was mounted parallel to the longitudinal axis of the model and with the probe body oriented at $45^{\circ}$ with respect 
to the $x$-axis (direction of free stream). A $6 \mathrm{~mm}$ cylindrical strut supported the hotwire assembly from the top; it was covered with an extruded aluminium airfoilshaped tube to minimize vibration and disturbance. The entire assembly was mounted on an $x-y$ traverse which rested on top of the test section. The dual-sensor probe consisted of two wires in an ' $\mathrm{X}$ ' configuration, each wire at $45^{\circ}$ with respect to the free stream, but perpendicular to its neighbour. The wires were of the same diameter as that of the single-sensor probe described above. The dual-sensor probe was mounted parallel to the free stream such that the ' $\mathrm{X}$ ' lay in the $x-y$ plane. In this orientation $u^{\prime}$ and $v^{\prime}$ fluctuations could be measured in the usual manner.

A two-channel constant-temperature anemometer circuit was built in-house by D. Nosenchuck, S. Taylor \& H. Robey of Caltech. Some modifications to the circuit were required to obtain a very high signal-to-noise ratio. Its output was analysed in one of two ways: $(a)$ for single-sensor real-time spectral analysis an HP $3582 \mathrm{~A}$ spectrum analyser was used, along with an $x-y$ plotter; $(b)$ for dual-sensor time-trace measurements, and for simultaneous hot-wire and smoke-wire measurements, a digital data-aequisition system was used. The anemometer output was sent to a signal conditioner which offset the d.c. component, amplified the signal to cover the full range of the $12-b i t A / D$ converter $(0-10 \mathrm{~V})$, and applied a low-pass filter as an anti-aliasing precaution. An 8-bit microcomputer was used to sample and record the data digitally on to floppy disk. At a programmable time during sampling, the smoke-wire could be turned on, as well as the camera and strobe, in order to record simultaneous hot-wire data and smoke-wire photographs. The signal conditioner and relay unit were built by one of the authors (J.M.C.); the microcomputer was also built in-house by D. Nosenchuck, S. Taylor \& H. Robey.

'The hot-wire data were subsequently analysed on GALCIT's DEC PDP-11 computer system.

\section{Flow-visualization results}

\subsection{Integration effect of streaklines}

Flow visualization, though a very useful tool in fluid-mechanics research, can often be misleading, as has been pointed out by Hooker (1936) and more recently by Hama (1962). The main problem with unsteady flows is that streaklines are not equivalent to streamlines, even though they are sometimes confused as such. In the laboratory, it is usually easier to generate streaklines than streamlines. The most common method of streakline visualization is the steady introduction of a flow tracer (typically dye or smoke) from a fixed location in the flow. For wakes this location is usually either upstream of the body, or on the surface of the body itself. But a dye or smoke filament is distorted as it travels downstream, and the streakline pattern seen at some downstream location contains information integrated all the way back to its point of introduction. Thus, when studying a streakline one cannot be sure if (a) the flow at a given location is currently experiencing the distortions implied by the streakline pattern, or $(b)$ that pattern is merely a remnant of the streakline's past history (an integrated 'memory' as it were). Therefore, in order to visualize the true nature of the flow at a given location, it is desirable to introduce the flow tracer close to that location. Traditionally the hydrogen-bubble technique in water has been useful because of its portability; the smoke-wire technique in air is analogous and has been employed here.

As a case in point consider the two-dimensional Kármán vortex street in the wake of a circular eylinder at low Reynolds number. Figure 1 shows a series of photographs 


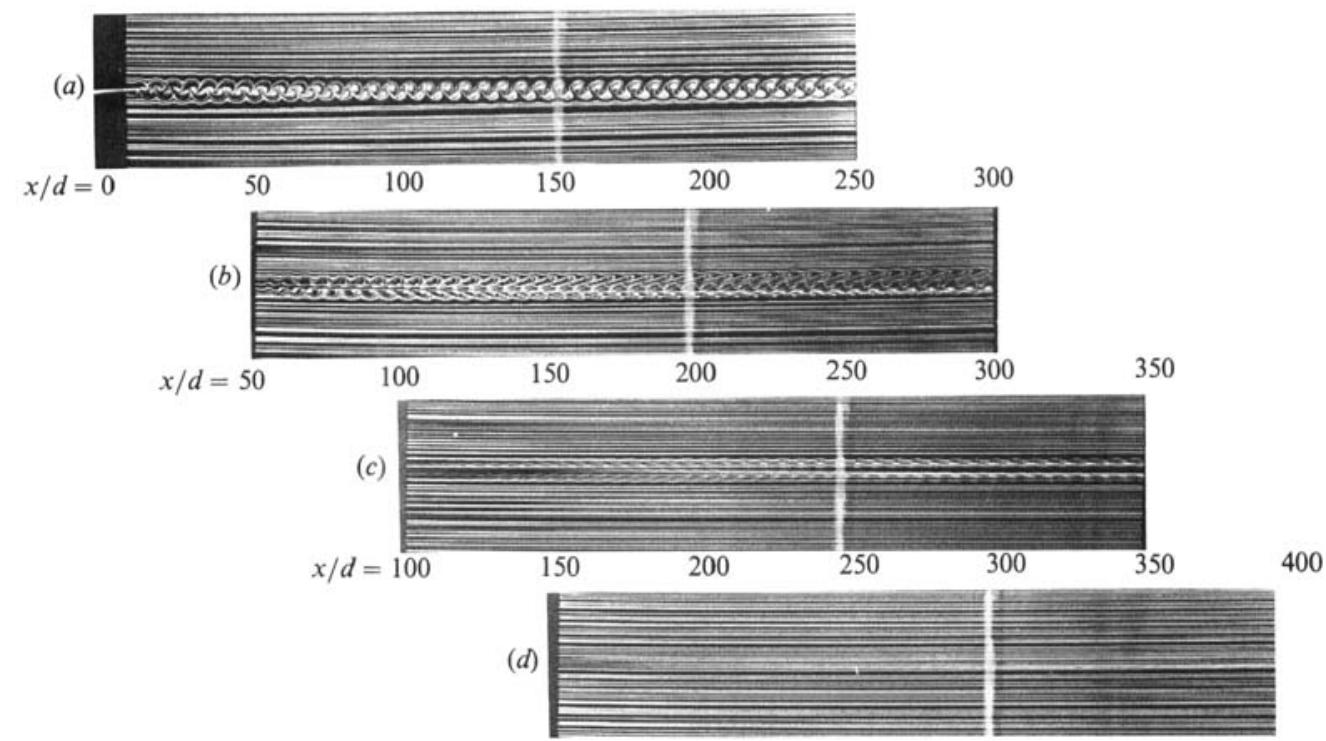

Figure 1. Circular-cylinder wake at $R e=90 ;$ smoke wire at $(a) x / d=4,(b) 50,(c) 100$ and (d) 150 .

taken at different times and at progressively increasing downstream locations in the wake at $R e=90$. The flow is from left to right, with the cylinder axis perpendicular to the plane of view. In each case the smoke wire has been placed at the left-most edge of the field of view, and the photographs are aligned so that any vertical line passes through the same downstream location in all of the photographs. In photo $(a)$ the smoke-wire is just downstream of the cylinder; the vortex street is clearly marked and remains visible to the downstream end of the photograph $(x / d=200)$. (The vertical white line at $x / d \approx 150$ is a rod supporting the smoke-wire assembly, and does not interfere with the flow - it is far out of the plane of smoke.) From $(a)$ alone one might conclude that the Kármán vortex street is still active at this downstream location. In $(b)$ and $(c)$ the smoke wire has been moved downstream to 50 and 100 diameters, respectively. The vortices are seen to be much weaker for these smoke-wire positions than would appear from $(a)$ alone. In $(d)$ the smoke wire is at $x / d=150$ and all the streaklines are parallel, which implies that the street at this location is so weak that it cannot be detected by the smoke-wire technique. This essentially parallel wake profile continues downstream with no further evidence of discrete vortices all the way to 350 diameters.

The integration effect of streaklines, as described above, is clearly demonstrated by comparing the flow pattern observed at $x / d=200$ in each of the photographs in figure 1 ; the pattern is drastically different, depending on smoke-wire position. Photo (a) shows what appears to be the Kármán vortex street at $x / d=200 ;(b)$ and $(c)$ show a similar structure but much weaker, while $(d)$ indicates that no structure is present at this wake location. Which photo then can we trust? That is, how can we objectively describe the flow field from this confusing array of photographs? It is our contention that in order to accurately discern the flow at some location, the smokewire must be placed at a proper distance upstream of that location. If it is placed too far upstream, the streaklines become very distorted and may lead us to erroneous conclusions. Likewise if it is placed too close to our observation point, the streaklines may not have had time to deform, again, causing confusion. 
The clue to understanding this figure lies in the following: Suppose a streakline rolls up as it marks a vortex moving downstream. The resulting pattern will clearly indicate that vortex. Now suppose that the vortex, as it travels, decays at a much faster rate than the smoke diffuses. We can then argue that the smoke remains in its integrated pattern, even after the vorticity has largely diffused (leaving an extremely weak vortex). The streakline pattern is now fixed, and is simply convecting along with the mean flow, no longer changing its shape. This is exactly what we observe in (a) of figure 1 ; notice that beyond 100 diameters or so, the streakline pattern no longer changes significantly - it merely convects downstream. There is of course some shearing of the pattern, as the outside of the wake convects faster than the centre. This can be seen in $(b)$ as well as in $(a)$ of figure 1. Zdravkovich $(1969)$ shows what he describes as extremely elongated elliptical vortices at $x / d \approx 600$; his smoke was introduced at the cylinder, and therefore suffers the same integration effect as our photo $(a)$.

Keeping these concepts in mind, we can now answer the questions posed above. Each photograph in the figure is useful only for a finite distance downstream of the smoke wire. To obtain an accurate description of the entire developing flow field, it is necessary to place the smoke wire at various positions, and then look at the collage of pietures as a whole. For example, the very rapid decay of the Kármán vortex street is not clear from any one of the photographs in figure 1; but when the entire figure is studied simultaneously, the decay is quite evident. We should mention here that the smoke wire itself has a wake, but the Reynolds number based on wire diameter is only about seven. At Reynolds numbers below forty or so, the wake of the smoke wire is steady and only influences the flow immediately downstream. We therefore consider the smoke wire as essentially non-intrusive.

At this point the reader may be questioning the validity of the above argument for air flow, where the ratio of viscous to molecular diffusivity (Schmidt number) is of order unity. One might argue that as a structure in the flow is dissipated by viscosity the smoke particles are diffused at nearly the same rate and smoke should not remain in a pattern for any longer time than the lifetime of the structure generating that pattern. A simple explanation concerning this apparent dilemma is as follows: Smoke in air is actually composed of tiny aerosol-type particles, whose mass is significantly greater than the mass of surrounding air molecules. Hence diffusion of these particles is extremely slow on a molecular scale; we may then define an 'effective Schmidt number' which is orders of magnitude larger than that for air alone. Lapple (1961) has calculated the diffusion coefficient $D$ of particulate matter into air at $25^{\circ} \mathrm{C}$. For oil smokes, particle size is approximately $0.2 \mu \mathrm{m}$, which gives a diffusion coefficient of about $2 \times 10^{-6} \mathrm{~cm}^{2} / \mathrm{s}$. In air, $\nu \approx 0.16 \mathrm{~cm}^{2} / \mathrm{s}$; our effective Schmidt number $v / D$ is thus of order $10^{5}$. That the diffusion of smoke is indeed slow is clearly seen in figure 1 ; if one follows a single streakline in the free stream outside of the wake, no significant dispersion of that streakline is observed (i.e. it does not broaden as it travels downstream).

\subsection{Decay of the Kármán vortex street}

As discussed above, an observation immediately apparent from figure 1 is the very rapid decay of the Kármán vortex street. This decay was found to occur at all the Reynolds numbers we investigated. In each case, when the smoke wire is placed far enough downstream of the cylinder, the shed vortices can no longer be found. Furthermore, the location of their disappearance seems to move upstream with Reynolds number. For $R e=90$ it occurs at about $125-150$ diameters. For $R e=155$, 
where the wake is just on the verge of transition to turbulent flow, the Kármán vortex street cannot be visualized beyond 75-100 diameters. (Of course, in this case a secondary wake structure is rapidly developing at this downstream location, as will be discussed in the following section.) For transitional and turbulent wakes $(R e>160)$ the vortices are dissipated much more rapidly, and are not seen beyond $x / d=50$.

The rapid decay of the Kármán vortex street has been known for quite some time. For example Roshko (1953) found that, for $R e=50$ and 100 , the energy intensity of the Kármán vortices is so low beyond 100 diameters that background tunnel turbulence becomes significant. For Reynolds numbers in the 'irregular' range $(150<R e<300)$, the decay is even more pronounced. For turbulent wakes Roshko's results are again supported by the present experiments, where Kármán vortices could not be seen beyond 50 diameters. Nevertheless, it seems that the full implication of this observed decay, particularly for laminar vortex streets, has not been appreciated owing to misinterpretation of streakline photographs. For example, Zdravkovich (1969) shows what appear to be very elongated Kármán vortices at $x / d=600$ ! In the light of our experience and the above discussion of streakline integration effects, these must be merely the integrated pattern from far upstream. Tritton (1977) states that '...the vortex street continues to all distances downstream,' again a misinterpretation of streakline patterns; in fact, the Kármán vortex street has completely decayed by at most one or two hundred diameters. Indeed, based on this rapid decay, the rows of vortices should perhaps not be called a 'street' at all (the original term having implied a long, regularly spaced pattern). In $\S 4$ we take a quantitative look at this decay, using hot-wire anemometry.

\subsection{The secondary vortex 'street'}

Because we are able to mount smoke wires at any desired position, the smoke-wire technique is convenient for studying development of downstream structure in a wake. In particular, placement of a smoke wire at positions beyond which the Kármán vortex street has largely decayed yields some interesting results. For Reynolds numbers less than about 100 we do not observe any further reorganization or development of large structure in the far wake. Figure 1 shows a typical case $(R e=90)$. Notice that beyond $x / d=150$, the streaklines in $(d)$ are parellel, with no apparent regions of concentrated vorticity. For these low-Reynolds-number wakes, large secondary structure could not be seen as far downstream as we investigated, which was about 500 diameters (not shown in figure 1).

For $100<R e<160$, a structure apparently similar to that of the Kármán vortex street, but of larger scale, is observed beyond a hundred diameters. Two cases in this Reynolds-number range are shown in figures 2 and 3 , at $R e=130$ and 155 respectively. The photos are aligned in the same way as described for figure 1 . The 'strength' of the secondary street may be inferred from how quickly the streaklines deform. From these photographs and others, we observe that the strength increases with Reynolds number in the range $100<R e<160$. (For example, the secondary structure in figure 3 at $R e=155$ is stronger than that at $R e=130$ in figure 2.) The scale of this structure is roughly 2-3 times that of the primary Kármán street and $a_{2} / a_{1}$ decreases with increasing $R e$ in this same range; $a_{2} / a_{1} \approx 2.3$ for $R e=130$, while $a_{2} / a_{1} \approx 2.1$ for $R e=150$. Figure 3 shows some of the strongest and most regular secondary structure that we have recorded on film. Photos (b) and (c) represent identical tunnel conditions, but were taken about $10 \mathrm{~s}$ apart. Both are included here to illustrate that the secondary structure is not a steady phenomenon-its 


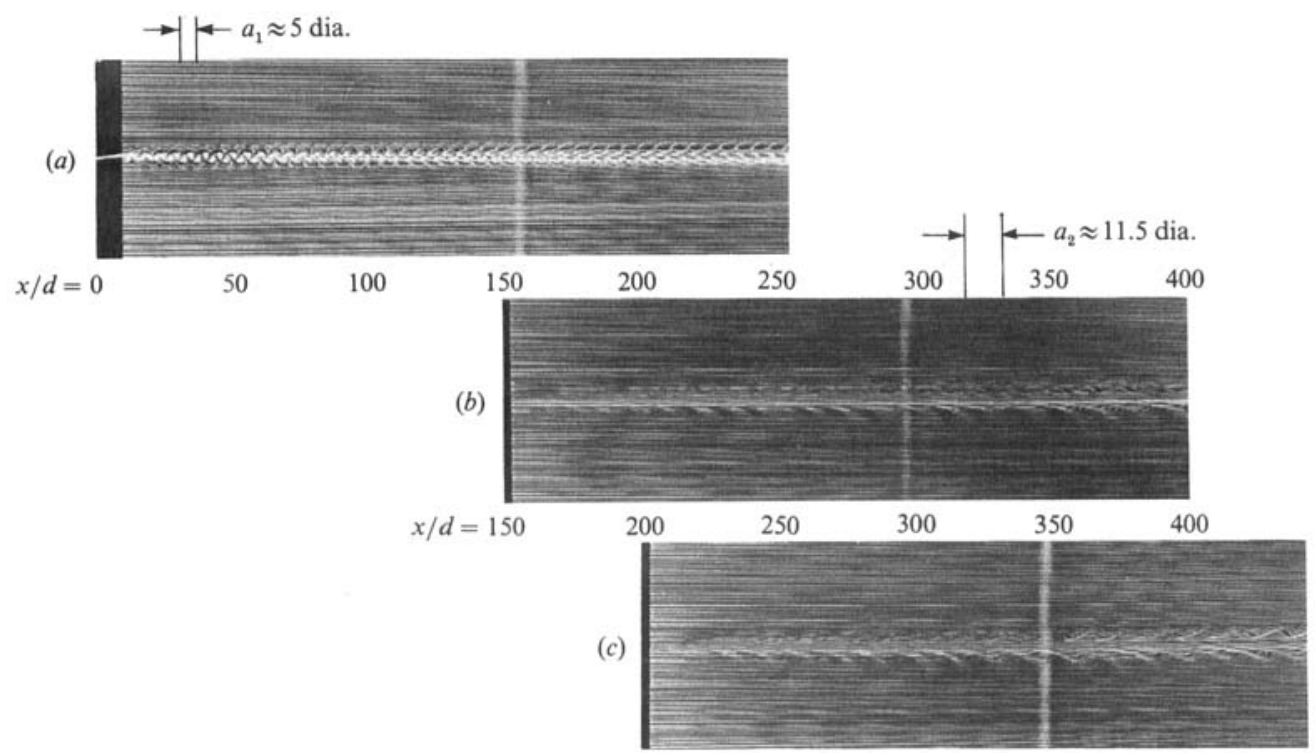

Figure 2. Circular-cylinder wake at $R e=130 ;$ smoke wire at $(a) x / d=8,(b) 150$ and $(c) 200$.

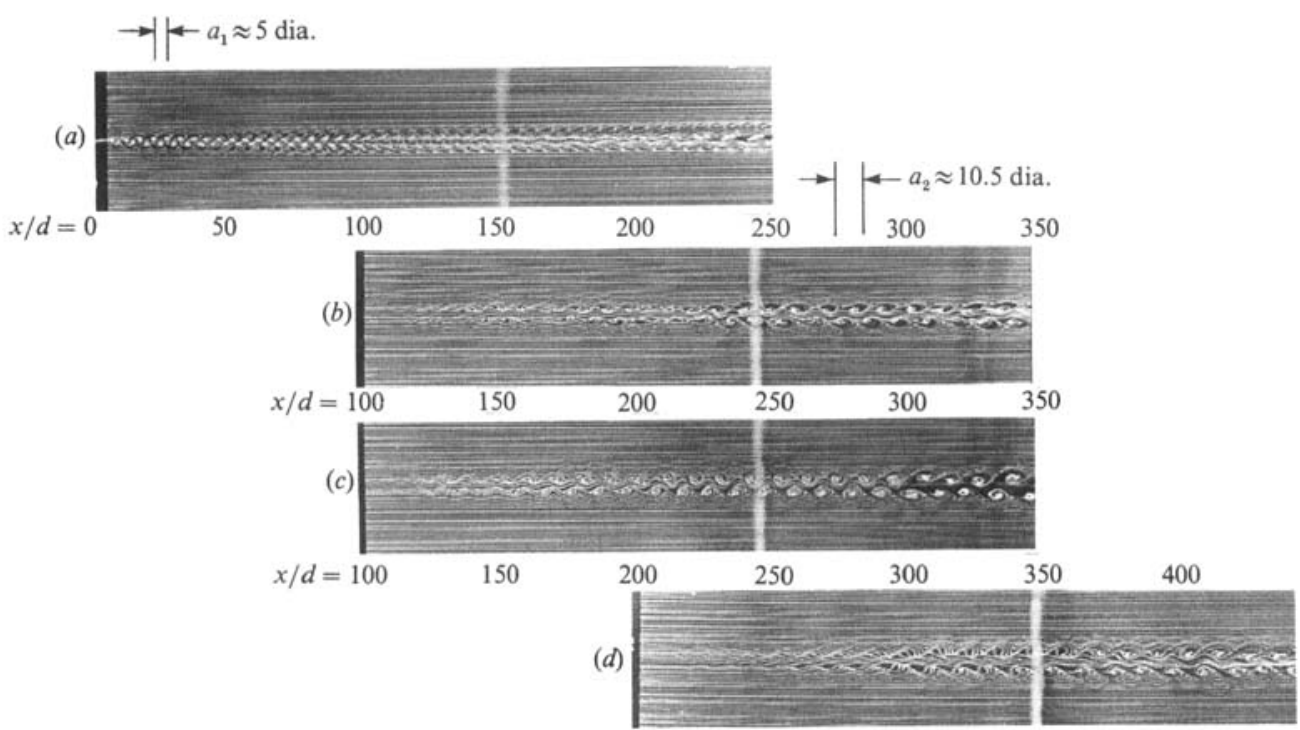

FiguRe 3. Circular-cylinder wake at $R e=155 ;$ smoke wire at $(a) x / d=8,(b) 100,(c) 100$ and (d) 200 .

appearance varies with time. Photo $(b)$ shows an irregular secondary street; this is a more typical case than $(c)$, where an unusually regular and strong street has been captured. Because the photographs shown here were not taken simultaneously, inconsistencies in the strength of the downstream structure may appear in collages like figures 2 and 3 . Three-dimensionality may play an important role in determining how regular the downstream structure appears in these edge views. Threedimensionality is discussed briefly in $\$ \mathbf{5}$.

For $R e>160$ the wake becomes irregular and eventually turbulent, making the interpretation of flow visualization extremely difficult. Taneda (1959) reported 

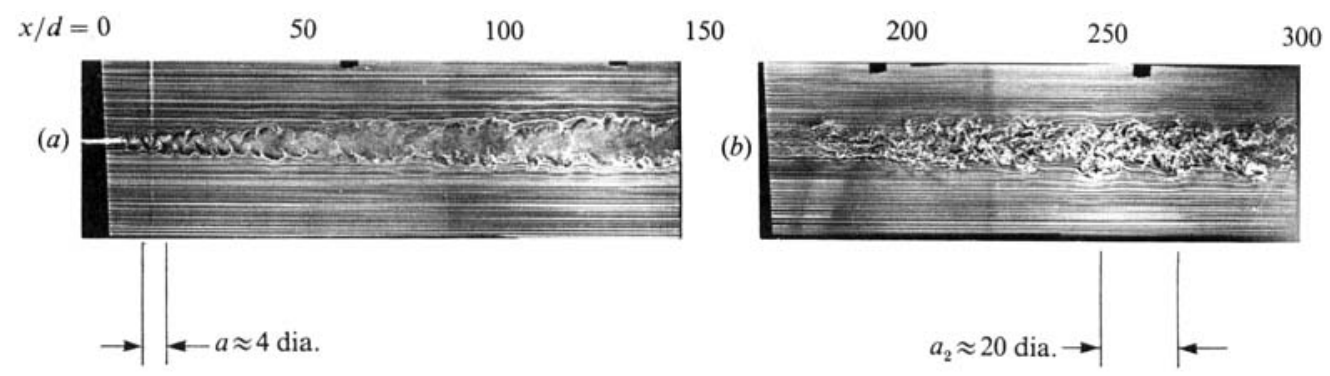

Figure 4 . Circular-cylinder wake at $R e=2200 ;$ smoke wire at $(a) x / d=1$ and $(b) 160$.

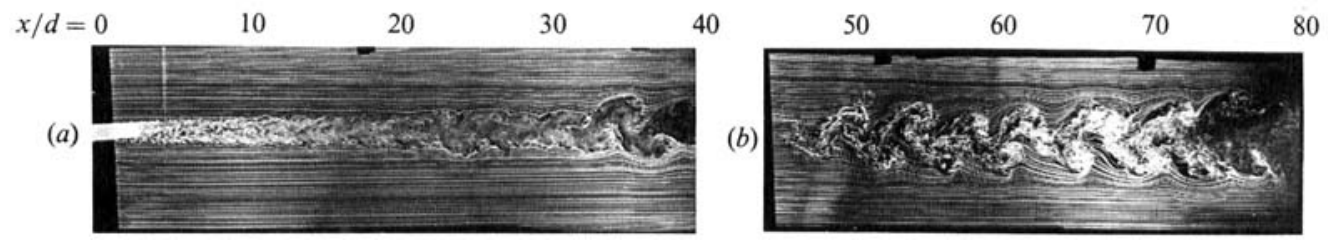

Figure 5. Porous-flat-plate wake at $R e=6000, \sigma=47 \%$ smoke wire at $(a) x / d=0$ and $(b) 44$.

seeing a secondary street at high Reynolds number, but only after several hundred diameters. Matsui \& Okude (1980) could not find any such street for $R e>160$, although they had not looked as far downstream as had Taneda. In the present experiments we did not find a well-organized, easily recognizable secondary 'street' for $R e>160$. However, 'groups' or 'bursts' of large vortical structures could sometimes be observed, similar to those observed by Grant (1958) and Townsend (1979). Figure 4, for example, shows a cylinder wake at $R e=2200$. Notice that after the Kármán street decays, the wake becomes very disorganized. However, beyond $x / d \approx 200$, (photo $b$ ) we notice a somewhat regular antisymmetric structure, of a scale much larger than the original Kármán street. The structure is most visible in the lower part of the wake at $x / d \approx 250$, where $a_{2} / a_{1}$ is about 5 .

\subsection{Porous-flat-plate wakes}

It is important to note than in all the above cases, secondary structure emerges after the primary (Kármán) street has largely decayed. The secondary structure therefore appears to develop independently; for example its scale (or frequency) is not necessarily in a fixed ratio to that of the primary street. This observation suggests that the secondary structure results from an instability of the mean wake profile. It therefore seems reasonable to assume that if one can produce a wake which initially has no Kármán vortex street, a street-like structure may emerge downstream owing to wake instability. Such a wake can be produced by a porous flat plate aligned normal to the flow direction, provided the solidity $\sigma$ is lower than about $80 \%$ (Castro 1971).

Smoke-wire photographs are shown in figure 5 for a plate with solidity $\sigma=47 \%$ at a Reynolds number of 6000 . The solidity is low enough that the plate does not shed Kármán-type vortices. The wake immediately downstream contains only small-scale structure, and has a 'top-hat' velocity profile in the mean. We do notice, however, a fairly regular vortex-street pattern at $x / d \approx 30$, presumably caused by far-wake instability. Let us re-emphasize here that the two photographs in figure 5 are not simultaneous, and therefore not necessarily consistent. It appears that photo $(a)$ 
was taken during a period of more intense structure than was $(b)$. It is likely that the initiation of the instability also fluctuates in the streamwise direction; the structure in (a) may have been initiated further upstream than that in $(b)$. At conditions similar to ours, Castro (1971) observed a dominant frequency in the wake, which he also attributed to hydrodynamic instability of the mean velocity profile. Valensi (1974) shows a similar photograph for a plate with $\sigma=53 \%$ at $R e=5 \times 10^{4}$. Hotwire measurements for the flow in figure 5 are discussed in $\$ 4$.

\section{Hot-wire measurements}

\subsection{Exponential decay of the Kármán vortex street}

Smoke-wire flow visualization of the far wakes of bluff bodies has given us new qualitative insight into Taneda's phenomenon of vortex-street breakdown and rearrangement. We now present the results of hot-wire measurements which give us quantitative information about this process. To obtain single-point velocity measurements in the wake, we used a 'cross-' or ' $X$-' wire, along with a digital dataacquisition system. The calibration procedure and details of the data acquisition are described by Cimbala (1984). Data were recorded at thirty-two $y$-locations for each of twelve streamwise positions in the wakes of circular cylinders at $R e=140,150$ and 500. Mean streamwise velocity profiles for the case $R e=500$ are shown in figure 6 . The least-square Gaussian fit to the velocity profiles is quite good beyond $x / d=100$, after the Kármán street has largely decayed; this has been shown more clearly by Desruelle (1983). Wake widths used in calculations in the following sections were taken from the Gaussian fits; for locations upstream of $x / d=100$ these give slightly smaller values for half-velocity width than indicated by the measured profiles.

First, let us consider the decay of the Kármán vortex street as downstream distance is increased. As had been pointed out by Bevilaqua (1975), velocity fluctuations due to a vortex street are more readily detected in the cross-wake $(v)$ velocity component. Hence, let us consider amplitude spectra of the $v^{\prime}$ fluctuations, where $v=V+v^{\prime}$. (An amplitude spectrum is the square root of a power spectrum; the units are in per cent of free-stream velocity). For a circular cylinder wake at $R e=150$ and at $x / d=25$ (our nearest measuring station), a delta-function spike is seen at the shedding frequency $f_{\mathbf{k}}$, which is the only significant frequency in the near wake. Proper characterization of changes in fluctuation magnitude with downstream distance is possible only when similar transverse locations in the wake are compared. For consistency, amplitude spectra were calculated at each $x$-location, averaged at the two $y$-locations corresponding to inflexion points in the mean velocity profile (one on either side of the wake). Because the inflexion point should be near the point of maximum mean shear, fluctuations in the transverse directions $\left(v^{\prime}\right)$ are expected to be highest near that point. Discrete spectral amplitude at $f=f_{\mathrm{k}}$ is plotted against downstream distance $x / d$ in figure 7 for $R e=140$ and 150. For both Reynolds numbers, the amplitude is seen to decay exponentially for $25<x / d<150$. By least-squares fitting a straight line through the first 6 or 7 data points in figure 7 , we obtain initial decay rates of 0.0246 decades per diameter for $R e=150$, and 0.0249 decades per diameter for $R e=140$. Similar decay rates have been reported for the $u^{\prime}$ fluctuations by other investigators. Hussain \& Ramjee (1976) obtained hot-wire traces of $u^{\prime}$ at $R e=145$ for various downstream distances. From their figure 4, the amplitude of the fluctuations can be seen to decay exponentially in the region $5<x / d<60$. In our earlier work (Cimbala et al. 1981) at $R e=155$, we measured discrete $u^{\prime}$ fluctuations at the shedding frequency, and found 


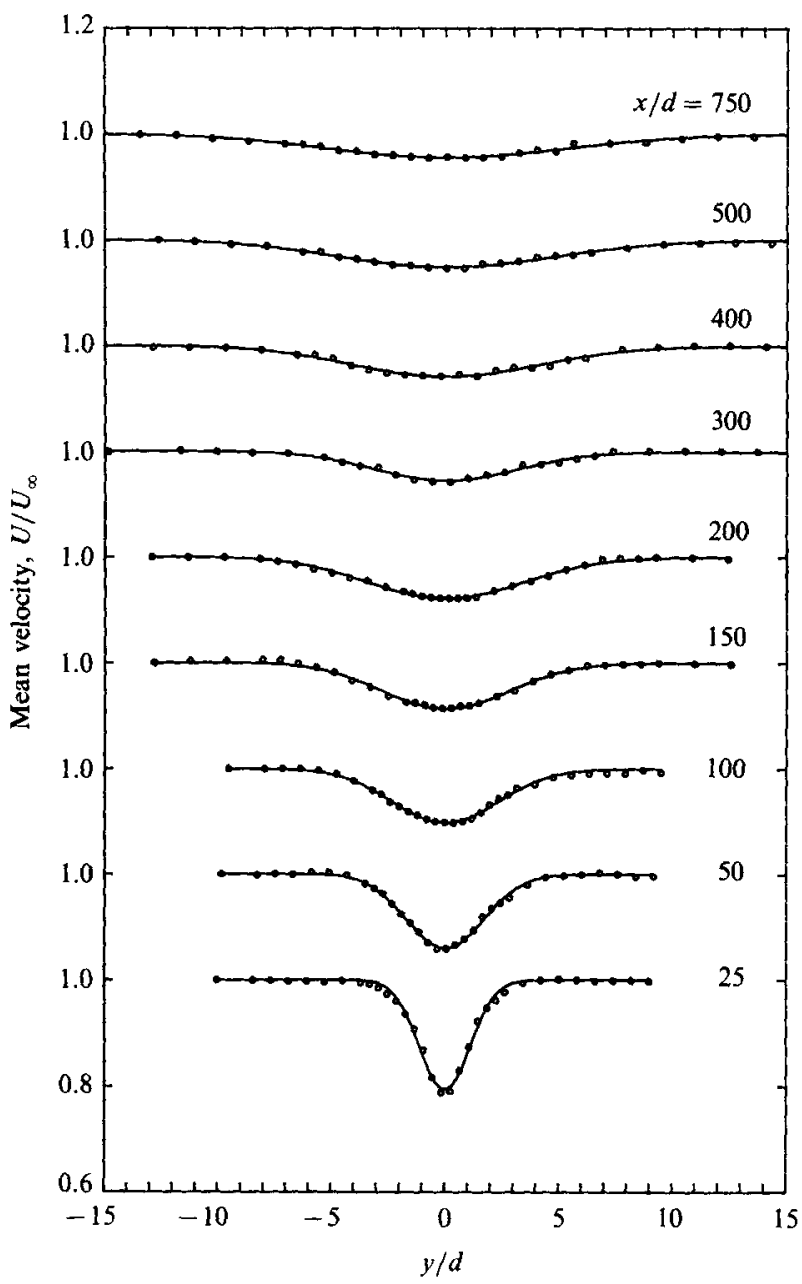

Figure 6. Mean velocity profiles for circular-cylinder wake at $R e=500 ; d=0.16 \mathrm{~cm}$, $U_{\infty}=4.97 \mathrm{~m} / \mathrm{s}$.

\section{Reference}

Desruelle (1983)

Desruelle (1983)

Present results

Present results

Matsui \& Okude (1981)

Hussain \& Ramjee (1976)

Present results

Present results

Desruelle (1983)

Cimbala et al. (1981)

Present results

Present results

$\begin{array}{crr}\text { Velocity } & R e & \begin{array}{r}\text { Decay ra } \\ \text { (decade/diam }\end{array} \\ u^{\prime} & 80 & 0.0143 \\ u^{\prime} & 120 & 0.0169 \\ u^{\prime} & 140 & 0.0817 \\ v^{\prime} & 140 & 0.0249 \\ u^{\prime} & 140 & 0.0170 \\ u^{\prime} & 145 & 0.0156 \\ u^{\prime} & 150 & 0.0209 \\ v^{\prime} & 150 & 0.0246 \\ u^{\prime} & 155 & 0.0225 \\ u^{\prime} & 156 & 0.0167 \\ u^{\prime} & 500 & 0.0284 \\ v^{\prime} & 500 & 0.0305\end{array}$

TABLE 1. Decay rates for velocity fluctuations measured by various investigators. 


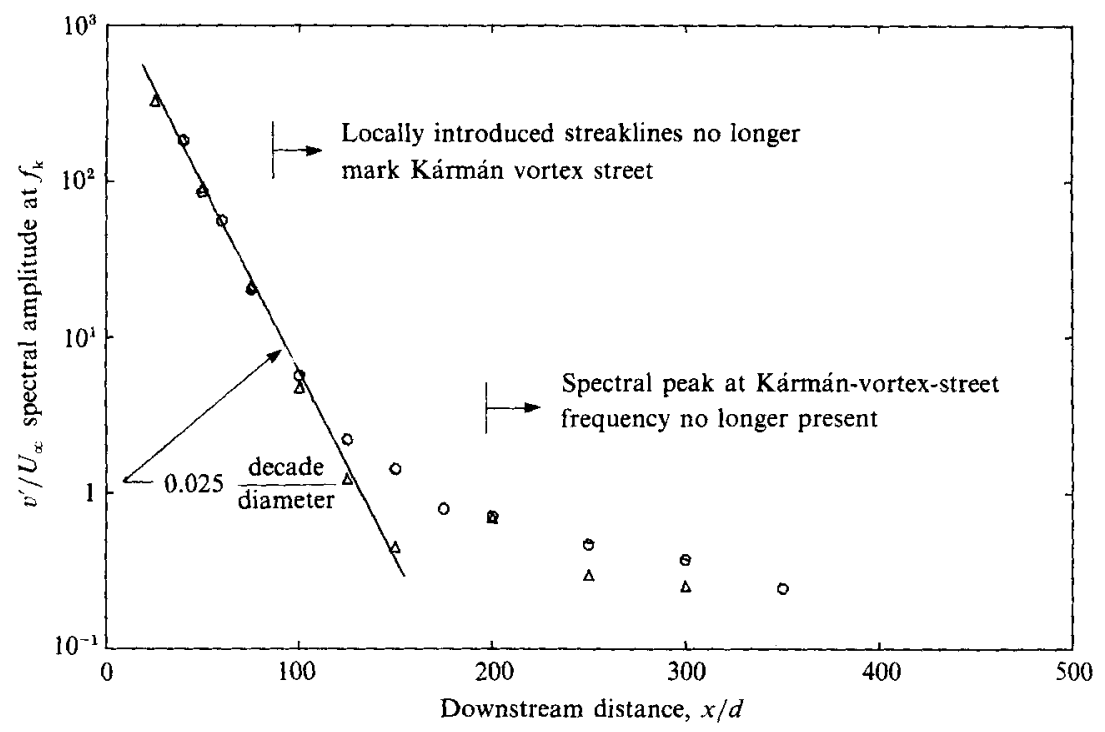

FigURE 7. Exponential decay of Kármán vortex street; circular-cylinder wake at $R e=140$ (O) and $150(\triangle)$.

$u_{r m s}^{\prime}$ to decay exponentially up to 100 diameters. Matsui \& Okude (1981) made similar measurements at $R e=140$, while Desruelle (1983) showed an exponential decay for three different Reynolds numbers. The decay rates for all these cases are summarized in table 1.

The general trend is for faster decay rates with increasing Reynolds number. This is especially apparent when we only consider data from one facility (e.g. the three rates of Desruelle, or our present results). There is some scatter in the data from different facilities; the decay rate is probably sensitive to background disturbances in the free stream. Since, as discussed in what follows, we believe that wake instability, even at Reynolds numbers as low as $10^{2}$, is primarily inviscid, this effect of Re seems puzzling at first. The explanation, we believe, is that the effect is an indirect one, the role of Re being mainly to change the initial wake conditions, through its effect on the separation point and the boundary-layer thickness, and thus on the wake profile.

\subsection{Downstream structure}

As downstream distance in the wake is increased, fluctuations appear at frequencies lower than the shedding frequency. Figure 8 shows amplitude spectra at several downstream positions for $R e=150$. Again the spectra are averaged over the two $y$-locations where the slope of the mean velocity profile is a maximum (inflexion points). We observe in figure 8 a shift to lower frequencies as $x / d$ is increased. In particular, the Kármán frequency $\left(f_{\mathrm{k}} \approx 166 \mathrm{~Hz}\right)$ is initially the only significant frequency, represented here by a delta function. The spectral amplitude measured at $x / d=25$ for example is several hundred, but decays exponentially as shown in figure 7. At $x / d=100$ in figure 8 a broad band of frequencies has arisen with the most prominent peak at $f \approx 90 \mathrm{~Hz}$, and other peaks at $f \approx 120,83$ and $70 \mathrm{~Hz}$. By $x / d=200$, disturbances at $f \approx 90$ and $70 \mathrm{~Hz}$ have amplified significantly, as well as the subharmonic $(f=83 \mathrm{~Hz})$; the peaks at $f=f_{\mathrm{k}}=166 \mathrm{~Hz}$ and at $f=120 \mathrm{~Hz}$ have decayed. With further increase in $x / d$, the band of prominent disturbances shifts to lower frequencies and broadens. At $x / d=400$ the most prominent band of frequencies 


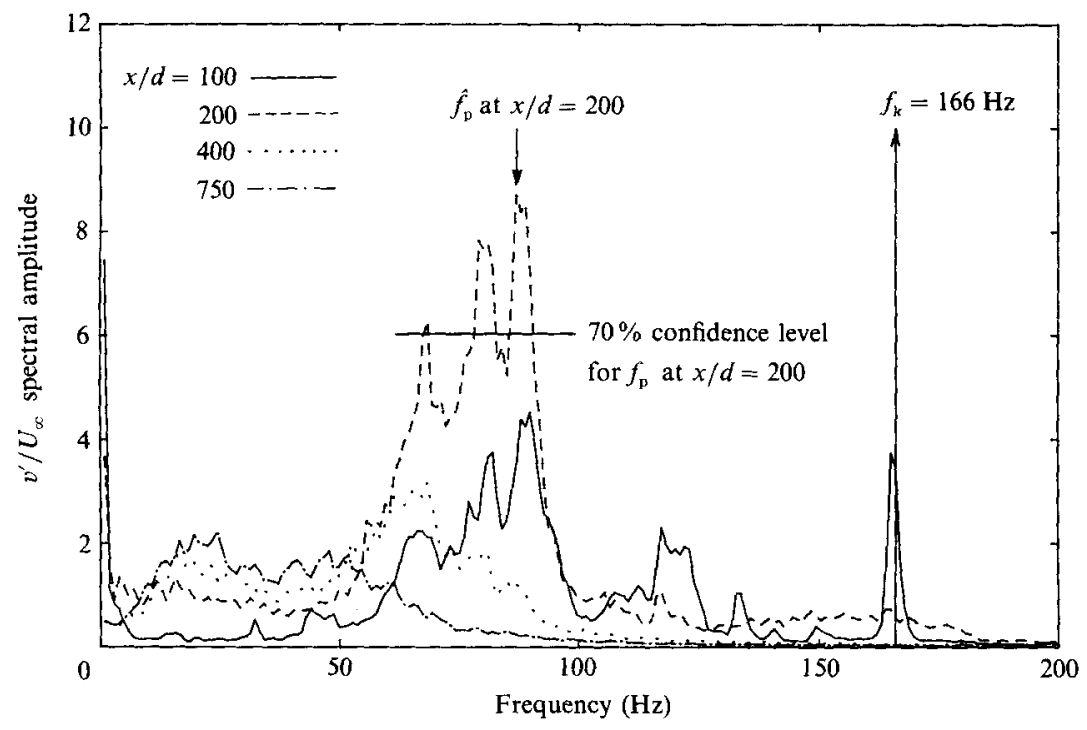

Figure 8. Amplitude spectra at several downstream distances; circular-cylinder wake at $R e=150$.

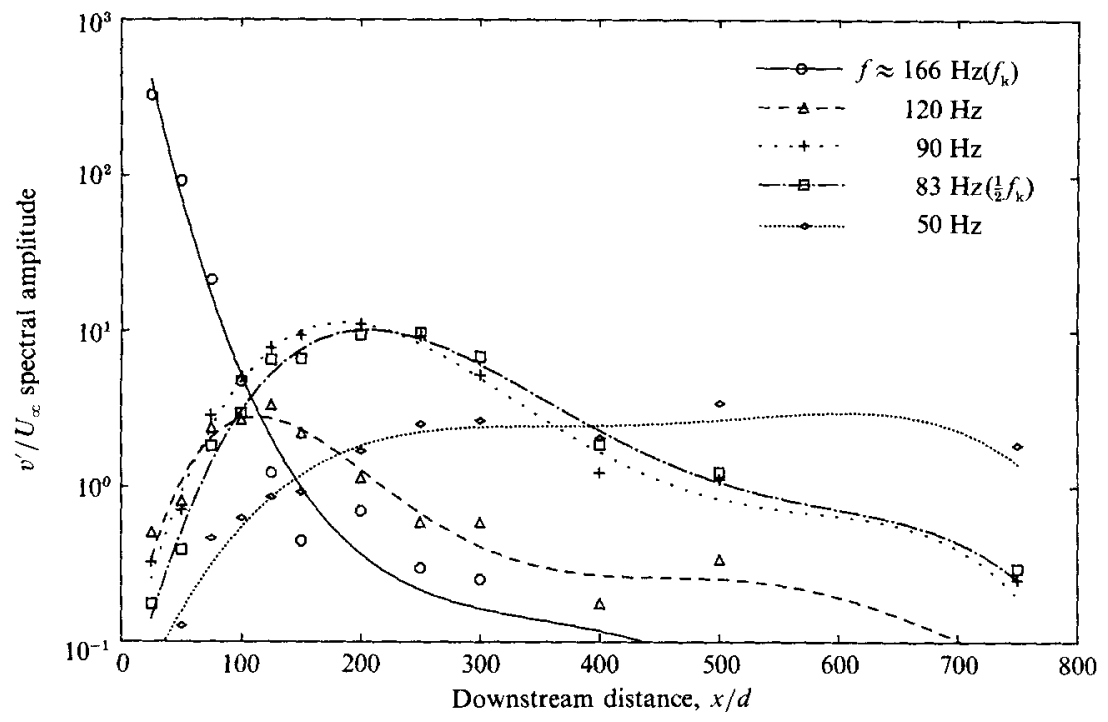

Figcre 9. Growth and decay of selected discrete frequencies; circular-cylinder wake at $R e=150$.

is centred around $70 \mathrm{~Hz}$, while all frequencies above $100 \mathrm{~Hz}$ have disappeared. By $x / d=750$, there are no longer any outstanding spikes; instead, a very broad hump is seen for $0<f<75 \mathrm{~Hz}$. In general, following the life cycle of a particular frequency, we notice that the fluctuation amplitude at that frequency first rises, then decays; the lower the frequency, the further downstream is its life cycle. In figure 9 we have plotted spectral amplitudes at several discrete frequencies versus downstream distance. The growth-decay cycle is clear.

Notice in figure 9 that there is nothing outstanding about the subharmonic, $f=83 \mathrm{~Hz}$. That is to say, disturbances at the subharmonic experience the same kind of growth and decay as at any other nearby frequency. For example, the growth- 


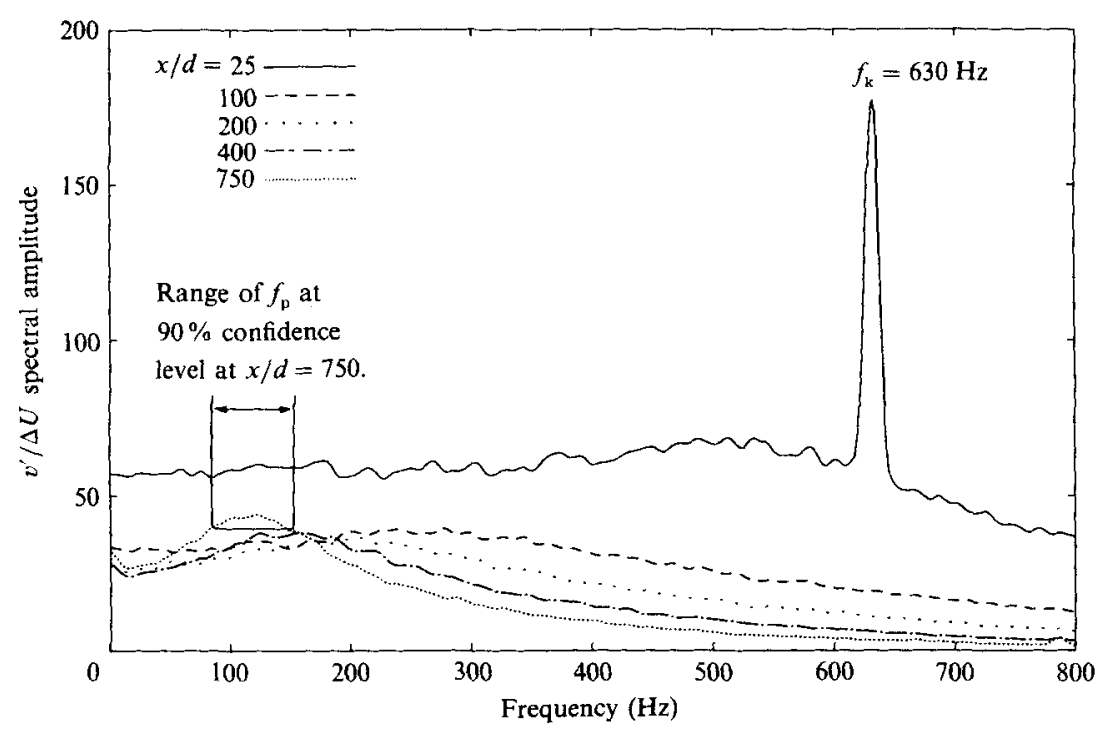

FigURe 10. Amplitude spectra at several downstream distances; circular-cylinder wake at $R e=500$.

decay cycle at $f \approx 90 \mathrm{~Hz}$ leads the one at the subharmonic by about 20 diameters, but is almost identical in magnitude and shape.

Figure 10 shows amplitude spectra at a cylinder Reynolds number of 500 , where the wake is turbulent. In this case there are no spectral peaks other than the one at the shedding frequency; instead a broad hump is discernible, which shifts to lower frequencies much more smoothly than for the laminar case above. The spectral amplitude of $v^{\prime} / U_{\infty}$ decays with downstream distance for all frequencies; i.e. we do not really see regions of growth, but rather decay, at each discrete frequency in these coordinates. In the spectra of figure $10 v^{\prime}$ is divided by the local mean velocity defect $\Delta U$, where $\Delta U=U_{\infty}-U_{0}=U_{\infty} W_{0}$, instead of by $U_{\infty}$. Frequency may also be normalized in the usual manner; i.e.

$$
\beta=\frac{2 \pi \delta f}{U_{\infty}}
$$

where $f$ is the dimensional frequency, and $\delta$ is the wake half-width, defined by the $y$-location where $U^{*}=0.5 ; U^{*}$ is the normalized mean velocity,

$$
U^{*}=\frac{U-U_{\infty}}{U_{0}-U_{\infty}}
$$

and $U_{0}$ is the mean centreline velocity. $U_{0}$ and the wake half-width $\delta$ are calculated by fitting a Gaussian profile to the measured mean streamwise velocities. In figure 11 we show normalized spectra at all twelve downstreams positions. These spectra show a self-similar behaviour beyond about 100 diameters. The centre of the spectral peak in these coordinates occurs at approximately $\beta=1.5$. The band of prominent frequencies is very wide, much more so than for the case of a plane mixing layer; namely $\Delta \beta / \beta_{\rho} \approx 0.7$ and 2.0 respectively where $\Delta \beta$ is the bandwidth at half the amplitude of the peak value at $\beta_{\rho}$. This is most likely due to the slower growth of wakes compared with mixing layers, which have growth rates $\mathrm{d} \delta / \mathrm{d} x=\frac{1}{2} \delta / x$ and $\delta / x$ respectively. The slower divergence of the wake permits a longer period in which 


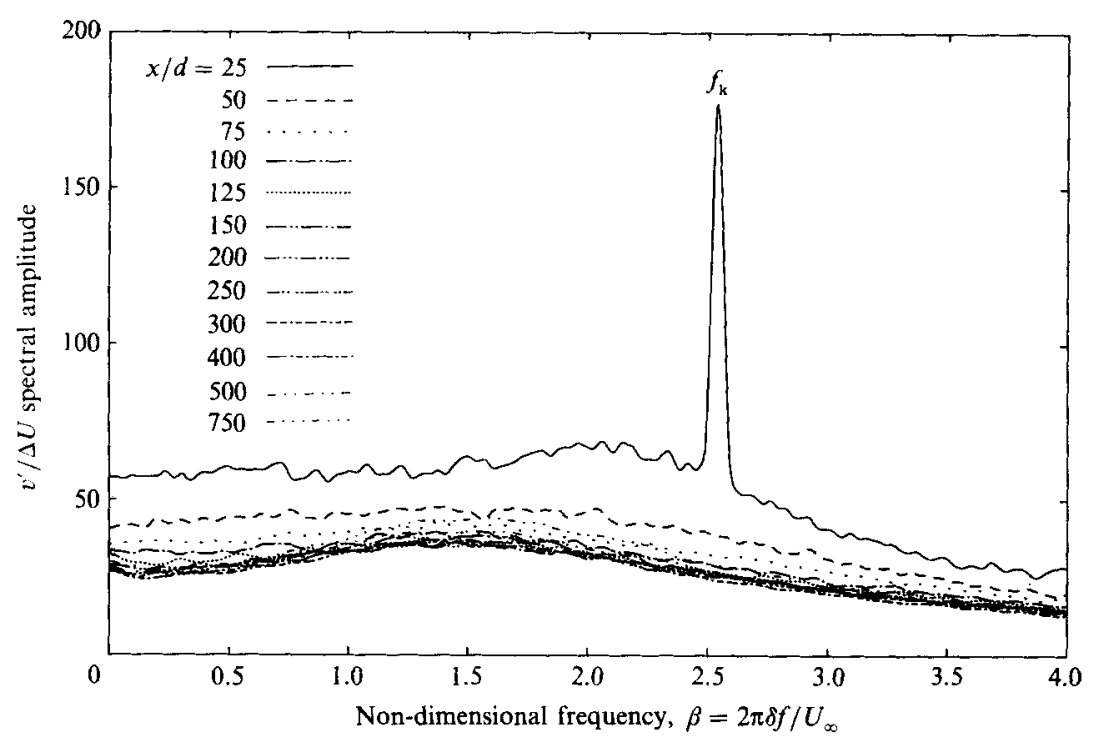

Figure 11. Normalized amplitude spectra at several downstream distances; circular-cylinder wake at $R e=500$.

unstable disturbances may grow. In the next section, we compare these results with predictions of linear stability theory.

\subsection{Comparison with linear stability theory}

Inviscid linear stability of a parallel wake profile has been analysed by many investigators, both in the temporal and spatial modes. Our intent here is to compare predictions from these analyses with our experiment results. Namely, for the measured mean velocity profiles, how well can linear theory predict which frequencies are most prominent in the wake?

For comparison with experiment, spatial stability analysis is preferred over temporal analysis, as has been pointed out by Mattingly \& Criminale (1972). T. Kubota, (1983, private communication) has generated numerical solutions for both cases; inviscid disturbances are superposed on a parallel Gaussian wake profile, details of which can be found in Cimbala (1984). Figure 12 shows spatial growth rate $g$ as a function of dimensionless frequency $\beta$, and for several values of $W_{0}$, the normalized centreline velocity defect in the wake $\left(W_{0}=1-U_{0} / U_{\infty}\right)$. Recall that for spatial stability analysis, frequency $\beta$ is real, while wavenumber $\alpha$ may be complex. The family of curves shown in figure 12 are for the temporal mode, where $\alpha$ is real and $\beta$ may be complex. We, however, plot spatial growth rate $g$, using the transformation of Gaster (1965). The important thing to note in the figure is the dependence of the growth curve on $W_{0}$. As we travel downstream in the wake, $W_{0}$ decreases, and the relevant growth curve is continually shifting toward higher $\beta$, as well as decaying in magnitude. For the case of a plane mixing layer, the velocity difference is constant, and there is only one growth curve, rather than a family of curves as in figure 12 .

T. Kubota (1983, private communication) has argued that the growth curves shown in figure 12 may be extended to negative growth (de-amplification) beyond the neutral point, $\beta_{\mathrm{n}}$. This is extremely important in the following analysis, where such a de-amplification region is required in order to predict growth-decay cycles of 


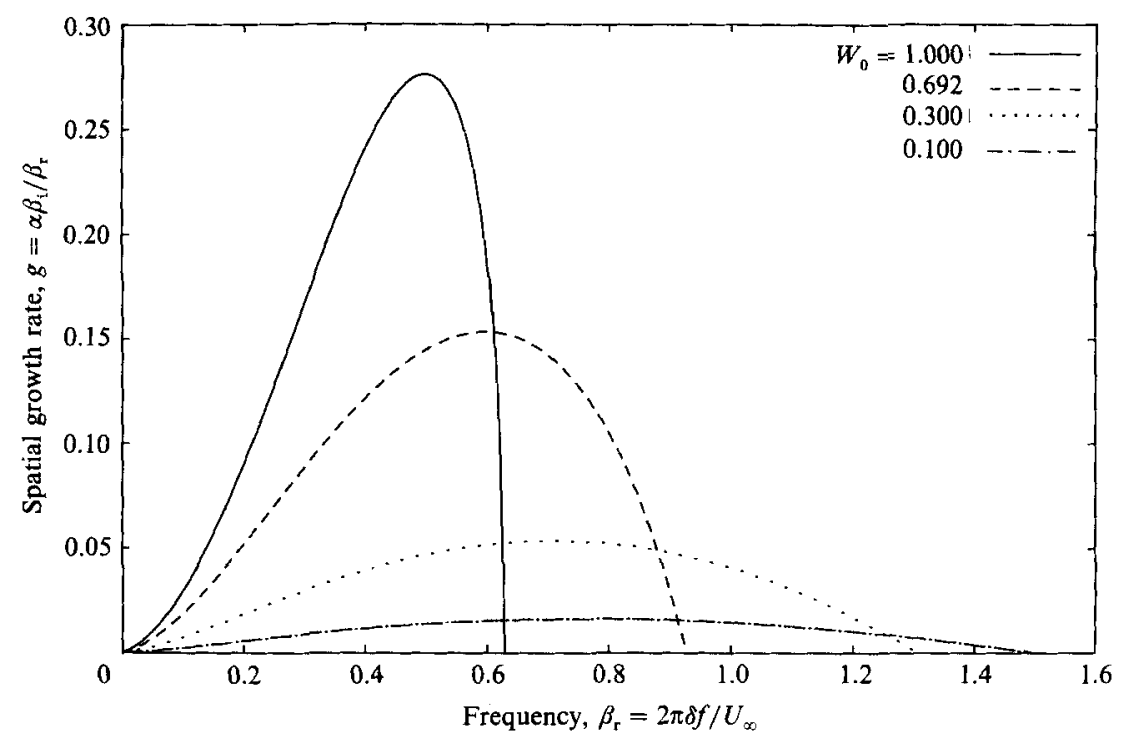

Figure 12. Amplification curves from temporal inviscid stability theory for wakes of decreasing velocity deficit.

large-scale structures. For lack of details in this region, we have extrapolated the curves in figure 12 by straight lines, whose slopes are matched with those at the neutral points. This extrapolation is obviously not entirely accurate, but reasonable; the predictions that follow would be affected somewhat by an alternate extrapolation.

Let us consider which prominent frequency $f_{\mathrm{p}}$ we expect to find in our measurements; i.e. we wish to predict $f_{\mathrm{p}}$ as a function of downstream distance $x$. At first glance one may expect $f_{\mathrm{p}}$ to equal the locally most-amplified frequency (the peak in the growth curve). This turns out not to be the case however, owing to nonparallelism of the mean wake. It must be remembered that the growth rates shown in figure 12 are calculated for a parallel wake (no $x$-dependence); the real wake of course is not parallel, but widens downstream. Obviously the best predictions would come from fully non-parallel stability analysis, where one takes downstream widening of the mean wake into account; nevertheless we expect the curves in figure 12 to be adequate approximations locally. Hence, a scheme was developed to approximate non-parallelism ( $x$-dependence) using only parallel stability calculations. Namely, in order to predict how large a disturbance to expect at some particular frequency, we integrate growth rate at that frequency with respect to downstream distance $x$. Doing this for a number of frequencies gives us disturbance amplitude as a function of frequency for each downstream distance. It is then a simple matter to find that frequency predicted to be the most prominent, $f_{p}$, as a function of $x$ (i.e. the frequency whose amplitude is greatest at a given $x$-location). This locally parallel scheme is described in more detail by Cimbala (1984).

Starting conditions for the locally parallel approximation are crucial, and unfortunately not easily chosen objectively. As mentioned previously, the velocity profiles upstream of about 100 diameters are not truly Gaussian, so the stability calculations of figure 12 do not strictly apply. We must start our integration upstream of this region, however, since there will be amplification of disturbances. The following simplifications have been applied; $(a)$ an initially flat spectrum 


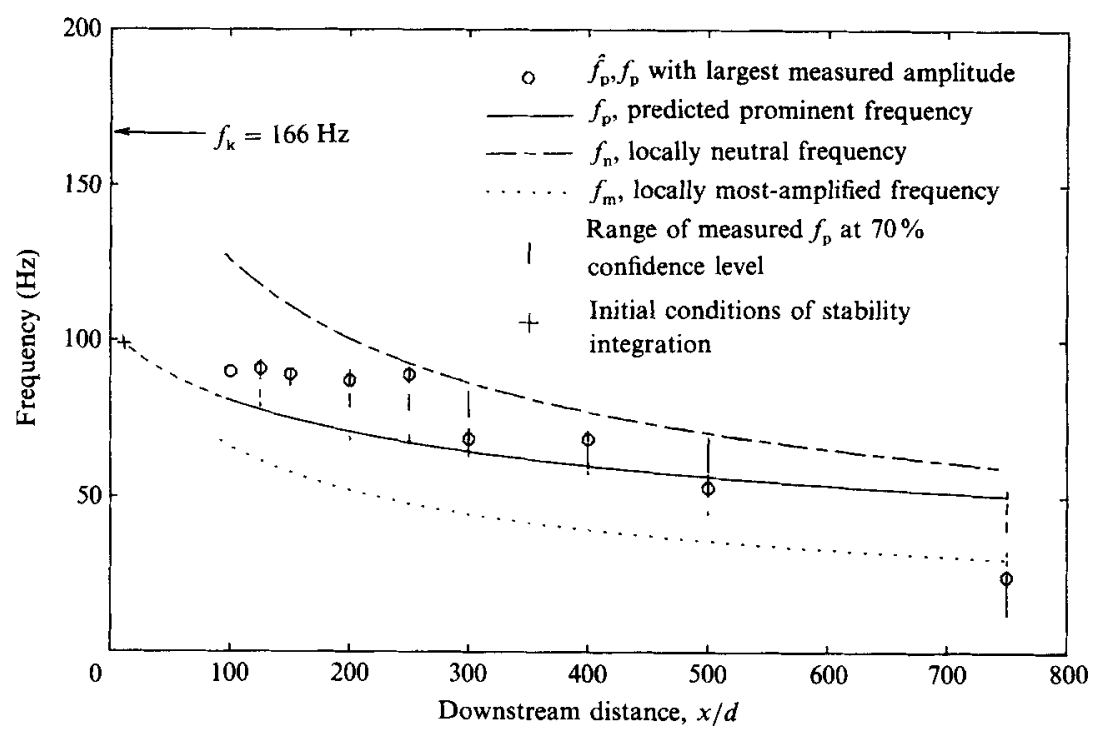

Figure 13. Prominent frequency versus downstream distance; circular-cylinder wake at $R e=150$.

(constant amplitude at all frequencies) is assumed; $(b)$ the growth rates of figure 12 are used, with $W_{0}(X)$ and $\delta(x)$ determined by least-squares Gaussian fits of the measured mean velocity profiles; $(c)$ integration is started at $x / d=10$, where $W_{0}$ and $\delta$ are extrapolated from farther downstream (our first measurement station is at $x / d=25$ ). Changes in any of these assumptions can change the predictions considerably. The reader should be aware that these calculations are only approximate since the actual wake is unsteady, non-Gaussian, non-parallel, threedimensional, and viscous. Our prediction scheme, based on inviscid, two-dimensional parallel stability of purely Gaussian wake profiles, should be considered a first attempt until more rigorous analysis is performed.

These predictions, which we are about to compare with the experiments, are based on two-dimensional, linearized theory. Within the linearized restrictions, this would not be affected by the presence of a three-dimensional (spanwise) linearized instability, even if the latter were comparable in strength with the two-dimensional one. We point this out because of what might seem an inconsistency in the application of two-dimensional results to the far wake where, as we shall see, the structure tends to be strongly three-dimensional.

Let us now compare our experimental results at $R e=150$ with predictions of inviscid linear stability theory. We show in figure 13 a plot of frequency versus $x / d$. The circles represent experimental frequencies $\hat{f}_{\mathrm{p}}$ at which the most prominent disturbances were measured. The vertical lines represent $70 \%$ confidence levels (as defined in figure 8), and give an idea of the bandwidth of prominent frequencies. These observed $f_{\mathrm{p}}$ should be compared to the predicted $f_{\mathrm{p}}$ as calculated with the locally parallel scheme - given here as the solid line. Also shown in figure 13 are the locally-neutral frequency (dashed line), and the frequency corresponding to the locally-most-amplified disturbance (dotted line). Note also that, because of the integration, the frequency that is locally most amplified is not necessarily the frequency with the largest (integrated) amplitude.

Close to the cylinder, the Kármán frequency $\left(f_{\mathrm{k}}=166 \mathrm{~Hz}\right.$, Strouhal number $S t=0.175$ ) is the most prominent observed frequency, as expected. In the very near 
wake, where the amplitude of fluctuations can exceed $30 \%$, comparison with linearized theory is not appropriate (Nishioka \& Sato 1978). Note that the Kármán vortices are initially formed by a mechanism much different from far-wake instability. The mechanism consists of a complex combination of unsteady boundarylayer separation and near-wake instability, involving also the body, and is not yet completely understood. We therefore do not expect $f_{\mathrm{k}}$ to match our predictions. Indeed, it is interesting in figure 13 that the Kármán frequency is well above our predicted prominent frequency. In fact, $f_{k}$ even lies above the locally neutral frequency $f_{\mathrm{n}}$, and is thus in the region of damping, according to stability theory. This is consistent with our observation that fluctuations at Kármán frequency $f_{\mathrm{k}}$ decay exponentially in the region $25<x / d<125$. This has also been verified in excitation experiments by Desruelle (1983).

As discussed above, several assumptions about initial conditions were necessary to obtain the predictions shown in figure 13. Changes in any of these assumptions would have an effect on $f_{\mathrm{p}}$. For example, starting the integration at a different $x$-location has the effect of moving the prediction curve $f_{\mathrm{p}}$ up or down, but the general trend of the curve remains the same. Because the near wake $(x / d<100)$ still contains large fluctuation amplitudes at the shedding frequency, the predictions in this region are not reliable. For this reason, a broken line on the $f_{\mathrm{p}}$ curve is used to show extrapolation upstream of the region of validity to the point of initiation at 10 diameters. (The region of validity begins at $x / d \approx 100$, beyond which the initial Kármán vortex street has largely decayed.) Figure 13 illustrates that after disturbances at $f_{\mathrm{k}}$ decay, lower frequencies take over; in the region of validity (beyond $x / d=100$ ), the agreement between predicted and observed frequencies is quite good.

What figure 13 suggests is that a prominent frequency stays constant until locally neutral conditions are reached, then drops to the predicted curve and again stays constant up to locally neutral conditions. The point at $x / d=750$ may be anomalous. For $R e=150$, such frequency plateaux seem consistent with the pictures. For $R e=500$ the continuously shifting spectrum suggests that the situation is much more volatile; i.e. at a given position $x$ a continuously changing history of scales is observed. It is significant to note that the maximum value of the broad spectrum of figure 11 occurs near $\beta=1.5$, which is close to the neutral stability point for the farwake profiles, as shown in figure 12 .

At Reynolds numbers above about 160 , the character of the wake changes considerably; by $R e=500$ it is 'fully turbulent'. Figure 14 shows the comparison between theory and experiment for the case of a turbulent cylinder wake at $R e=500$. Here $f_{\mathrm{k}}$ is approximately $630 \mathrm{~Hz}$ and is off the scale. In this case $f_{\mathrm{k}}$ is prominent only in the very near wake. Already by $x / d=50$, a lower-frequency band (centred around $f_{\mathrm{p}} \approx 290 \mathrm{~Hz}$ ) is prominent. The observed $f_{\mathrm{p}}$ decreases quite smoothly with $x$ as we travel downstream. $90 \%$ confidence levels have been chosen as defined in figure 10 ; the band of prominent frequencies is very broad. For this turbulent case, our observed $f_{\mathrm{p}}$ lies above the predicted $f_{\mathrm{p}}$; in fact, it follows more closely the locally neutral frequency $f_{n}$. This indicates that the initial conditions assumed in the locally parallel approximation as described above, may not be as appropriate for this case. (The initial conditions here are consistent with those assumed for the laminar case.) There are several differences between the turbulent and the laminar wake which should be considered in choosing initial conditions. (a) There are more disturbances available in the turbulent near wake - our assumption of an initially flat spectrum may not be adequate. $(b)$ Integration should be started immediately downstream of the cylinder, rather than at $x / d=10$. This would have the effect of shifting our 


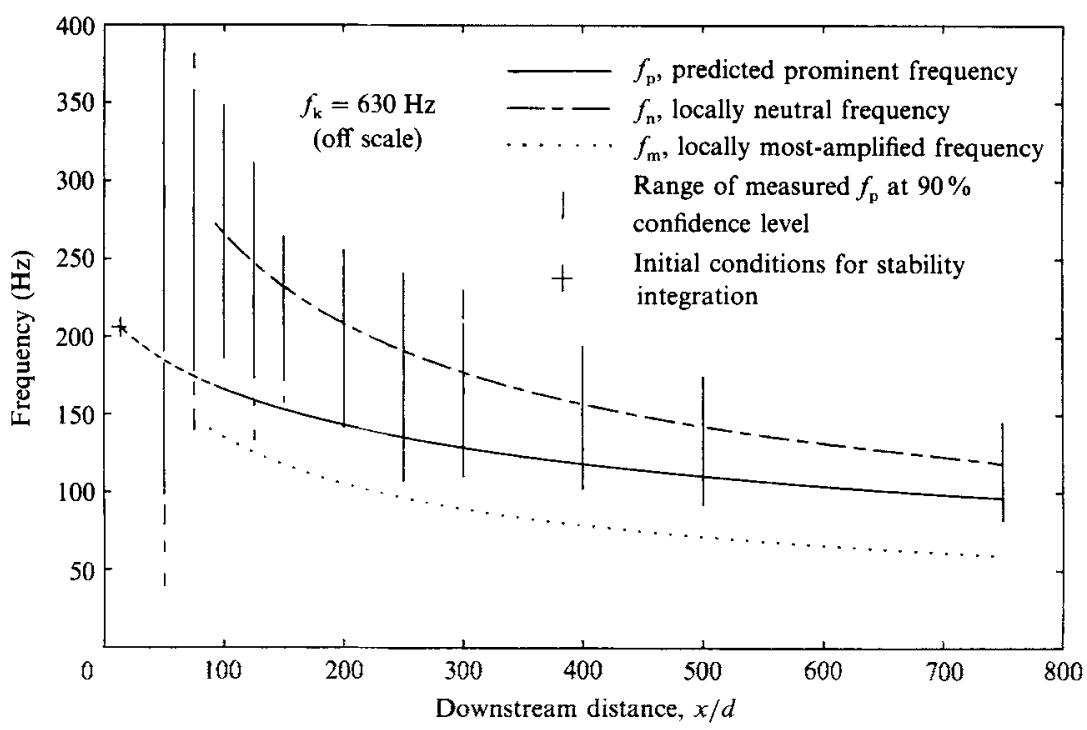

Figure 14. Prominent frequency versus downstream distance; circular-cylinder wake at $R e=500$.

prediction curve upwards. However, extrapolations to this region would be extremely unreliable and were not attempted. $(c)$ The shed Kármán vortices in the case of the turbulent wake decay more quickly, allowing other modes to develop earlier - again the effect would be an upward shift of $f_{\mathrm{p}}$. (d) Finally, small-scale three-dimensional fluctuations are present in the turbulent wake, which may affect stability; our calculations are strictly two-dimensional. The unsteady nature of the near-wake region dominated by the shed Kármán vortices must be kept in mind in interpreting these estimates based on linear stability theory of a steady velocity profile.

Figure 14 indicates that for quick and practical estimates, one may use the locally neutral curve to predict prominent frequencies in a turbulent far wake. We may point out here that similar results have been reported recently by Wygnanski et al. (1986) for the case of a plane turbulent wake at $R e=4000$; namely, the observed prominent frequency matches well with the neutrally amplified frequency, as predicted by local linear stability theory. Ho \& Huerre (1984) have observed this agreement for the case of a plane turbulent mixing layer as well. These observations are related to Lessen's marginal stability model, which actually makes a somewhat different statement (Lessen \& Singh 1974).

It is encouraging (and somewhat surprising!) that such a simple prediction scheme as employed here works as well as it does, considering that the actual flow is nonlinear, unsteady, non-parallel, and (as will be discussed below) three-dimensional. Furthermore, the presence of large-scale vortices indicates that the disturbances never really see the time-averaged profile upon which our stability analysis is based. Nevertheless, for reasons not entirely understood, we may conclude from this exercise that two-dimensional inviscid parallel hydrodynamic stability theory, when interpreted properly, is adequate to estimate the most-probable frequencies in a far wake. Furthermore, this supports the hypothesis that structure appearing far downstream of a bluff body is the result of hydrodynamic instability of the velocity profile, and is not directly dependent on the shed vortices of the Kármán vortex street. 


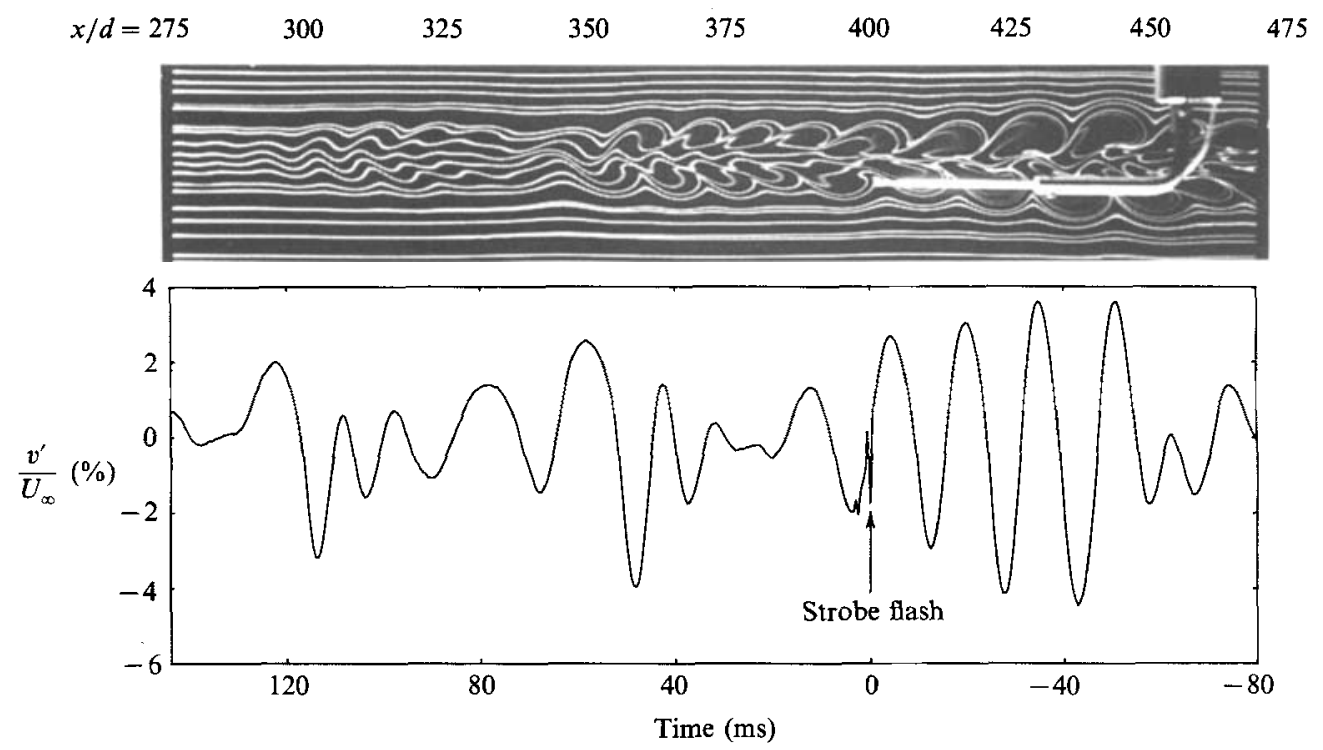

Figure 15. Simultaneous smoke-wire and cross-wire records, circular-cylinder wake at $R e=150$; smoke-wire at $x / d=275$, cross-wire at $x / d=400, y / d=4$.

\subsection{Simultaneous smoke-wire and cross-wire records}

The microprocessor was programmed so that a smoke-wire photograph could be taken at any specified time during the data-collection interval. This enabled us to display smoke-wire and hot-wire records simultaneously; an example is shown in figure 15 for a cylinder wake at $R e=150$. The smoke-wire is at $x / d=275$, while the cross-wire is positioned at $x / d=400$ and at $y / d=-4$. Note that in order to avoid damage to the hot wires, the cross-wire assembly was moved about $1 \mathrm{~cm}(\approx 8$ diameters) out of the plane of smoke. For comparison, at $x / d=425$ the vortex spacing is about 13 diameters. The time trace in figure 15 has been displayed 'backwards' (i.e. time increasing to the left), so that direct comparison between the flow-visualization photograph and time trace is possible. The scales have been matched according to

$$
t=\frac{x}{U_{\mathrm{c}}}
$$

where $U_{\mathrm{c}}$ is the local convection velocity (assumed to be the mean velocity at the location of the probe). In this example, $d=0.0016 \mathrm{~m}, U_{\infty}=1.528 \mathrm{~m} / \mathrm{s}$ and $U_{\mathrm{c}}=1.397 \mathrm{~m} / \mathrm{s}$. The time axis has also been shifted so that the strobe flash occurs at $t=0$.

Because of the integration effect of streaklines, as discussed in $\$ 3$, we do not expect the time trace to exactly match the distortions of streaklines in the photograph. Nevertheless, a general agreement is certainly present. Notice for example the group of orderly structures at $-60<t<0$; large-scale vortices are clearly seen in the photograph at $400<x / d<460$. Just ahead of the probe $(350<x / d<390)$ there appears in the photograph a group of smaller-scale vortices. This is also recognizable in the time trace as a very weak higher-frequency oscillation. Short-time spectra of the fluctuations shown in figure 15 give frequencies of $64 \mathrm{~Hz}$ for the larger-scale structure, and $84 \mathrm{~Hz}$ for the smaller-scale structure. Both of these frequencies are 


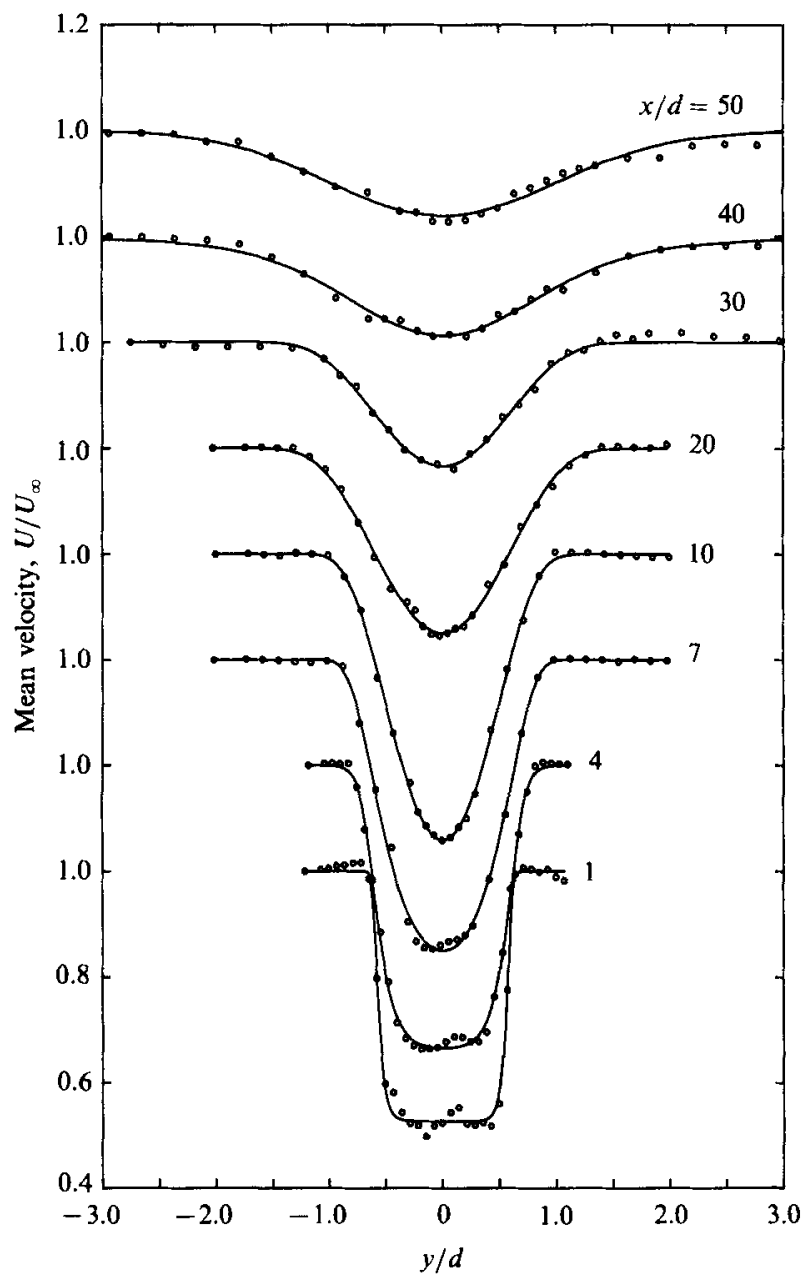

Figcre 16. Mean velocity profiles for porous flat-plate wake; $\sigma=47 \%, R e=5000 ; d=1.78 \mathrm{~cm}$,

$$
U_{\infty}=4.54 \mathrm{~m} / \mathrm{s} \text {. }
$$

within the range of amplified frequencies, according to linear stability theory, as can be seen in figure 13 at $x / d=400$.

Both the time trace and smoke-wire photograph of figure 15 support Townsend's suggestion that far-wake structure appears as groups of several vortices; this is consistent with the hypothesis that downstream structure develops from wake instability of the mean velocity profile and not directly from vortex amalgamation.

\subsection{Surveys of porous-flat-plate wakes}

Cross-wire surveys were also taken in the wake of a $47 \%$ solid flat plate at $R e_{d}=5000$, where $d$ is the width of the plate. Mean velocity profiles are shown in figure 16. As expected, very near to the plate the profile is a sharp 'top hat' which gradually relaxes as the shear layers on either side of the wake begin to merge. By ten diameters the profiles are far-wake-like, and have been Gaussian fitted here.

Amplitude spectra of $v^{\prime} / U_{\infty}$ are plotted in figure 17 for several $x$-locations; each spectrum is averaged at the two inflexion points of the mean velocity profile, as 


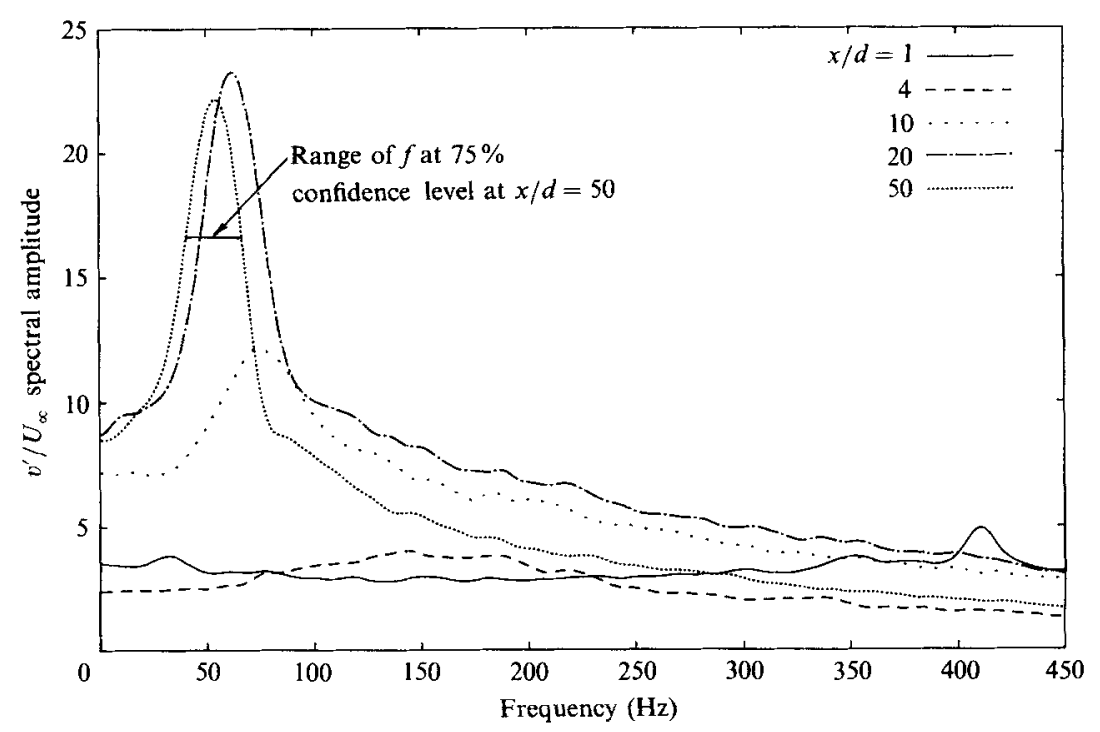

Figure 17. Amplitude spectra at several downstream distances; porous-flat-plate wake $(\sigma=47 \%)$ at $R e=5000$.

discussed previously. At $x / d=1$, spectral energy at low frequencies is small, but there is a noticeable bump centered around $410 \mathrm{~Hz}$. Recall from our flowvisualization results that the plate does not appear to shed Kármán-type vortices; the peak in the spectrum is associated with instabilities in the plane shear layers which exist initially on the outer edges of the wake. We can calculate the local Strouhal number of one of these shear layers;

$$
S t=\frac{f \theta_{\mathrm{SL}}}{\bar{U}}
$$

where $\theta_{\mathrm{SL}}$ is the shear-layer integral thickness,

$$
\theta_{\mathrm{SL}}=\int_{-\infty}^{\infty}\left(\frac{U-U_{1}}{U_{2}-U_{1}}\right)\left(1-\frac{U-U_{1}}{U_{2}-U_{1}}\right) \mathrm{d} y
$$

and $\bar{U}$ is the average of $U_{1}$ and $U_{2}$. At $x / d=1$ we have $U_{1}=2.39 \mathrm{~m} / \mathrm{s}, U_{2}=4.54$ $\mathrm{m} / \mathrm{s}$, and $\theta_{\mathrm{SL}}=0.0507 \mathrm{~cm}$, calculated from the data in figure 16 . For $f=410 \mathrm{~Hz}$, equation (4.4) gives $S t=0.06$, which is consistent with the observations of Ho \& Huerre (1984) for a growing free shear layer, that is $S t=0.032$ when the shear layer first begins to oscillate, and $S t=0.079$ further downstream as the shear layer grows. Our value lies between these two extremes, which supports our speculation that the peak at $f=410 \mathrm{~Hz}$ is associated with the shear layers on either side of the wake, and not with Kármán-vortex shedding. Recall that flow-visualization photographs also do not show a shed vortex street (figure 5).

At four diameters downstream the amplitude spectrum is devoid of any outstanding peaks; a very broad band is centred at approximately $150 \mathrm{~Hz}$ and the peak at $410 \mathrm{~Hz}$ has disappeared. We shall designate the range $4<x / d<10$ as a transition range, where the stability problem changes from that of two (independent?) plane shear layers to that of a plane far wake.

At $x / d=10$ the spectrum has increased in magnitude at the lower frequencies, and 


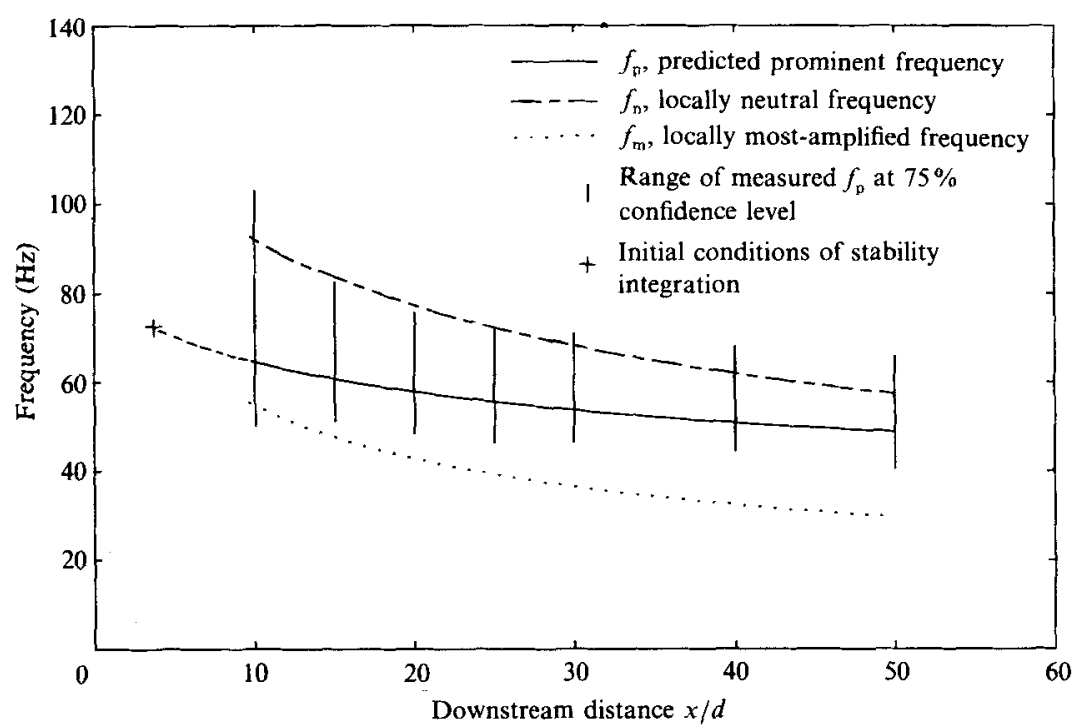

Figure 18. Prominent frequency versus downstream distance; porous-flat-plate wake $(\sigma=47 \%)$ at $\operatorname{Re}=\mathbf{5 0 0 0}$.

in particular there is a broad hump centred around $75 \mathrm{~Hz}$. Beyond 10 diameters, in the 'far-wake' region, the peak in the spectrum shifts to lower frequencies (larger scales) as downstream distance increases.

Figure 18 shows a comparison between observed and estimated prominent frequencies $f_{\mathrm{p}}$; the predicted values come from locally parallel stability analysis, as discussed above. Here, the wake is Gaussian-like beyond about 10 diameters. The integration was started with a flat spectrum at $x / d=4$, using extrapolated values of $W_{0}$ and $\delta$, in the manner described in $\$ 4.3$ for the case of a circular-cylinder wake. Again, changes in the initial conditions would move our prediction curve up or down within the bounds of $f_{\mathrm{n}}$ and $f_{\mathrm{m}}$ respectively, but the general trend would he retained. The agreement in figure 18 is quite good beyond $x / d=10$, in the far-wake region. $75 \%$ confidence levels are also plotted in figure 18 to indicate the bandwidth of prominent frequencies. The significance of these porous-plate data is this: hydrodynamic instability in a far wake leads to the growth of downstream structure, the scale of which is determined by local wake properties; the far-wake structure is not dependent on the scale of shed Kármán vortices - which for the case of a porous plate do not even exist.

\section{Three-dimensional effects}

Up to this point our discussion has been confined to the $x-y$ plane; now let us consider three-dimensionality. The earliest experiments on far-wake structure are those of Townsend (1956) and of Grant (1958). Grant's work, an extension of Townsend's, consists of long-time-averaged velocity correlations in the far wake ( $\approx 500$ diameters) of a two-dimensional cylinder at $R e=1300$. A peculiar threedimensional structure was inferred from these time-averaged measurements, and was dubbed the 'vortex-pair eddy', later called the 'double-roller eddy' by Townsend (1970). More detailed calculations by Payne \& Lumley (1967) of Grant's data yielded a similar structure. 


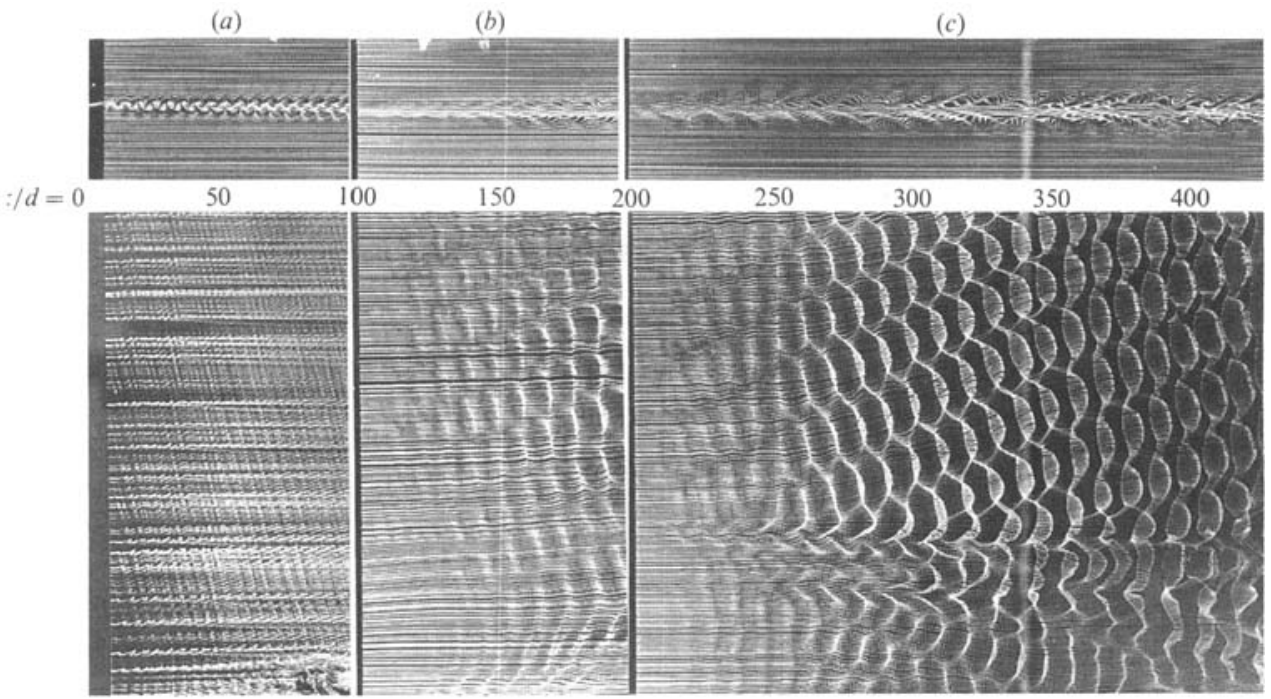

Figure 19. Edge and plan views of a circular-cylinder wake at $R e=140 ;$ smoke wire at (a) $x / d=8,(b) 100$ and $(c) 200$, and at $y / d=0$.

Since that time, neither the instantaneous nature of this structure nor its origin have been adequately explained, although there has been some speculation (see for example, Keffer 1965; Townsend 1970, 1979; Roshko 1976). Roshko (1976) suggested that the structure may be the time-averaged superposition of vortex loops, formed by the pinching off and joining together of vortices from opposite sides of the street. That long-time averaging seriously distorts one's interpretation of the instantaneous structure was also mentioned by Townsend (1979). He suggested that time averaging of the large-scale velocity patterns 'makes them appear more complex because of the superposition of patterns from eddies at all stages of ... [his proposed] growth-decayrenewal cycle'. An excellent discussion of some of these ideas is offered by Wlezien (1981).

Most recently Mumford (1983), using a pattern-recognition technique, suggests that the double-roller eddies may be even more complex than previously thought. He found that the structures are often confined to one side of the wake centreplane, rather than extending across the entire wake. Furthermore, eddies of similar type tend to occur in groups of two or more, one after another in the streamwise direction.

Whatever the exact nature of the far-wake large structure, various indications suggest that it is three-dimensional. To investigate this visually, the smoke-wire flow-visualization set-up was modified for plan views by aligning the cylinder parallel to the smoke wire. Figure 19 shows both edge $(x-y$ plane) and plan $(x-z$ plane $)$ views of the cylinder wake at $R e=140$. (The two views were recorded for the same tunnel conditions, though not simultaneously.) The edge view illustrates the decay of the Kármán vortex street and the subsequent growth of secondary structure. The smoke wire was positioned at $x / d=8,100$ and 200 in the manner discussed previously.

For the plan view, the smoke wire was at the same three $x$-positions as above, but at $y / d=0$ (i.e. in the centre plane of the wake), and parallel to the cylinder. In photo (a) one immediately notices the skewed angle at which vortices are shed from the body. The reason for this is not entirely clear, but similar observations have been reported by many others (see for example Tritton 1959; Gerrard 1966; Nishioka \& 


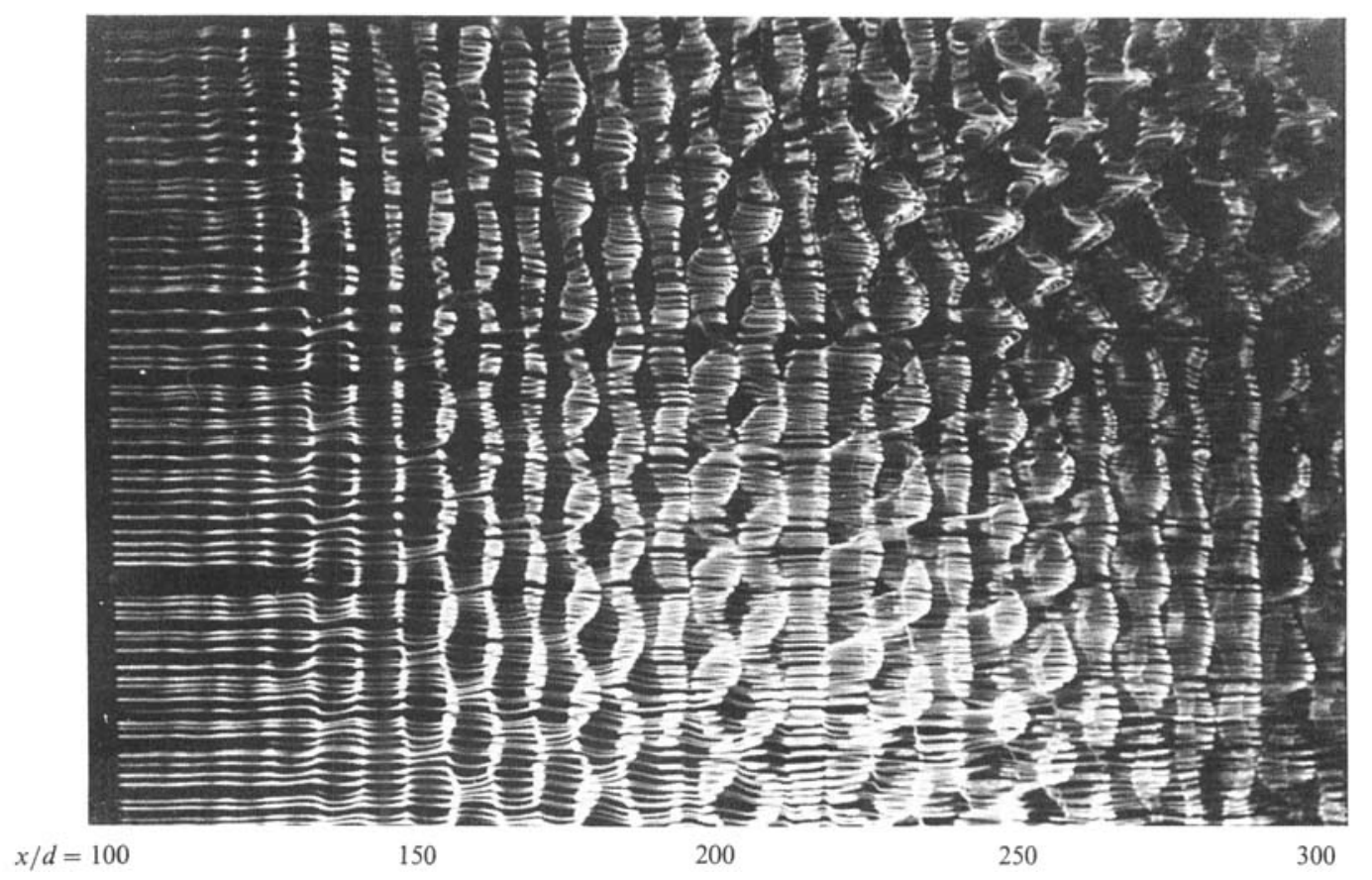

Figure 20. Plan view of a circular-cylinder wake at $R e=150$; smoke-wire at $x / d=100, y / d=2$.

Sato 1978). Our own experiments indicate the slantwise shedding to be quasi-stable; i.e. vortices are sometimes shed parallel, sometimes slanted one way, and other times the opposite way. Subtle non-uniformities of the free stream, end conditions, or the body itself are the most likely candidates for triggering transitions between the three 'modes'. Cimbala (1984) shows an unusual case where vortices are shed slantwise at two opposing angles at different parts of the span with an 'elbow' in between. Similar behaviour and its sensitivity to upstream and cylinder conditions was noted by Desruelle (1983). His experience indicates that slantwise shedding from a slightly inclined cylinder leads to more laterally uniform Kármán vortices.

In figure 19 the shed vortices are parallel to each other, with no appreciable spanwise structure. After the decay of the primary street however, threedimensionality is seen in the secondary structure beyond 100 diameters (photos $b$ and $c$ ). Lateral non-uniformities in the near wake may well act as disturbances in the development of these far-wake three-dimensional structures as noted by Desruelle (1983). The honeycomb-like pattern also is skewed in these photographs; but, just as with the shed vortices, the skewness comes and goes. The size of the cells is approximately 20 cylinder diameters. Our interpretation of the pattern in the plan view photograph $(c)$ is that it depicts only one side of the wake. This interpretation is based on additional evidence like that presented in figure 20 .

What exactly is this structure? What is the mechanism by which it develops? How, if at all, does it relate to Grant's 'vortex-pair eddy'?

Figure 20 provides additional information for a conjecture on the answers to these questions. For $R e=150$, with the smoke wire at $x / d=100$, we see the gradual formation of three-dimensional structure. By moving the smoke wire out of the wake centreplane $(y / d=2)$ we are able to visualize vortex lines from just one side of the secondary street. In this particular case, the vortices are initially straight and 


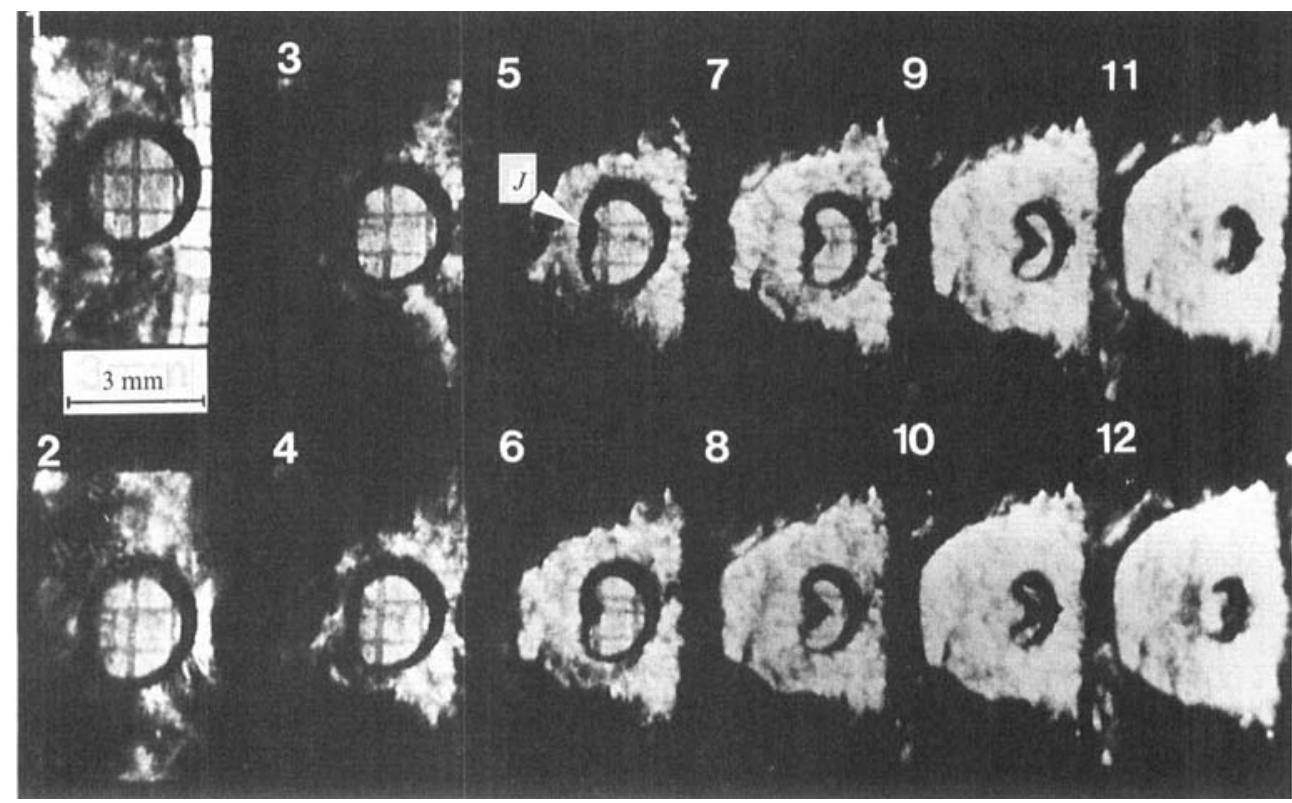

Figure 21. Edge and plan views of a circular-cylinder wake at $R e=190$; smoke wire at (a) $x / d=8,(b) 100$ and $(c) 200$, and at $y / d=0$.

parallel. A waviness quickly develops, with successive vortices $180^{\circ}$ out of phase. The amplitude of this perturbation grows downstream, eventually to where one vortex overlaps the next. This can most readily be seen in the upper right-hand corner of the photograph, where hairpin-line vortices, not unlike those observed in the transition process of boundary layers (see e.g. Saric \& Thomas 1984), are developed. Presumably the other side of the street has a similar experience.

Long-time-averaged correlations of such loops could be interpreted as Grant's 'vortex-pair eddies', that is, the Townsend-Grant large-scale structures are the legs of the hairpin vortices on each side of the wake. This description is consistent with Mumford's (1983) findings. Some evidence that vortex loops can exist in a wake was provided by Breidenthal (1980) who used a three-dimensionally perturbed splitter plate in a shear-layer facility to produce the loops, which persisted for large downstream distances.

Our observations, therefore, suggest that this three-dimensional structure is a result of a secondary instability based on the interaction of spanwise oblique (and non-uniform) disturbances with the two-dimensional periodic vorticity structures of the far-wake instability. This secondary instability is in the form of a parametric subharmonic resonance similar to that analysed by Pierrehumbert \& Widnall (1982) in free shear layers and more extensively for boundary layers and channel flows by Herbert $(1983,1984)$. In fact the symmetric profile of the Poiseuille flow (Herbert 1983 ) is the closest to the conditions in the wake.

For higher Reynolds numbers, where the wake becomes turbulent, smoke visualization is encumbered by small-scale structure. In figure 21 however, at $R e=190$, one can still recognize wavy structures similar to those in the laminar wake. 


\section{Discussion and conclusions}

\subsection{Decay of the Kármán vortex street}

We found smoke-wire flow visualization to be very effective, as well as easy to use, in the study of wakes of bluff bodies. We must emphasize, however, that extreme caution should be taken when interpreting streakline patterns in flows with any type of unsteadiness. In particular, when the classical case of a Kármán vortex street is visualized by placement of smoke wires at several downstream locations, the confusing integration effect of streaklines becomes immediately apparent. The conclusion to be drawn is that in an unsteady flow it is best to introduce flow tracers at not just one, but several positions in the flow. Only by studying a collage of photographs arranged in the manner described in this paper can one be confident of not being misled by the integration effect of streaklines.

It has been shown, with flow visualization and again with hot-wire measurements, that the Kármán vortex street shed in the wake of a circular cylinder does not persist indefinitely, but rather decays exponentially with downstream distance. In the present experiments, the decay is so rapid that fluctuating velocity measurements at the Kármán frequency are lost in background 'noise' by 100 to 150 diameters at $R e=150$. The decay is even faster for turbulent wakes where small-scale structure is prevalent.

Such rapid decay is by no means a new revelation; however its importance has not been realized up to now because of the misinterpretation of streakline flow visualization. Reasons for the decay are $(a)$ viscous diffusion, and $(b)$ de-amplification in the sense of hydrodynamic stability. Numerical streakline simulations of Cimbala (1984) included only the first of these, i.e. viscosity. The decay rate inferred from these simulations is not as rapid as that for the experimental case of figure 1 ; this shows that viscous diffusion is not the only mechanism of decay. Desruelle (1983) shows evidence that the second mechanism, hydrodynamic deamplification, accounts for at most $77 \%$ of the vortex-street decay rate. Other possibilities contributing to the decay include $(c)$ cancellation of vorticity from opposite sides of the street, and $(d)$ that the Kármán vortices may be located differently with respect to the centreline than a regular eigenfunction. This final point was suggested by Desruelle, where he shows that as $\delta$ increases with $x$, the lateral spacing $b$ between Kármán vortices does not 'keep up'; Kármán vortices that initially sit on the edge of the wake move closer to its centre as the wake grows. More detailed studies of this phenomenon and its effect on the decay of a vortex street have not been attempted to date.

\subsection{Development of donwstream structure}

As the wake widens with downstream distance, the scale of large structures must also increase. The mechanism by which this is accomplished is hydrodynamic instability of the developing wake profile. Frequencies are selectively amplified and then damped, according to the local growth rates, as determined by the local wake profile. Observed prominent frequencies agree fairly well with estimates of locally parallel inviscid linear stability theory, provided that streamwise growth of the wake is taken into account.

For unsteady laminar wakes, $100<R e<160$, the distribution of frequencies that are prominent broadens with downstream distance. The structure appears as groups of several vortices, separated by regions of random fluctuations; the frequency (or scale) of vortices within a group is constant, while it can vary considerably from group to group. Such a pattern agrees intuitively with what one expects in a 
hydrodynamically unstable system. As a disturbance grows it convects downstream until it is no longer amplified, but damped. Meanwhile, in somewhat random fashion, disturbances at other frequencies are amplified, perhaps originating at different $x$-positions, and go through similar life cycles.

The above scenario is appropriate for turbulent as well as laminar wakes. In the turbulent case, additional complications arise because of small-scale (threedimensional) turbulent structure. Townsend (1966) proposes his equilibrium hypothesis as a mechanism for the growth-decay cycles of large structures, namely, large eddies develop during a period of quiescence, when the turbulence intensity is low. The large eddies grow in strength and cause rapid entrainment, which leads to an increase of turbulence intensity at all scales. Turbulent motion of smaller scales resists the growth of large eddies by absorbing some of their energy. The large eddies therefore decay, and another period of quiescence begins. Further downstream, where the wake is wider, larger-scale (lower-frequency) structure emerges, and the cycle recurs. Our results are not inconsistent with Townsend's equilibrium hypothesis. We stress in addition the important role of local hydrodynamic stability in the growth and decay of large structures.

Let us now address the phenomenon of vortex amalgamation. As discussed in $§ 1$, there has been some difference in view as to the role of vortex pairing in the changeover to larger-scale structure in wakes. There are proposals that pairing instability is paramount in this changeover. It is our contention however that vortex amalgamation is not necessary for the growth of larger structure; but rather, hydrodynamic instability of the developing velocity profile results in the selective amplification of frequencies which are locally unstable. If amalgamation does occur it is purely incidental, not the driving mechanism of the flow. For our circularcylinder wakes at $R e=150$ and 500 , vortex pairing was not observed. Furthermore, we found nothing outstanding about the subharmonic frequency of the shed Kármán vortex street. That is to say, disturbances at the subharmonic do not experience a different history of growth and decay than other nearby frequencies. Wakes of porous flat plates provide further evidence that vortex amalgamation is not an essential mechanism for wake development. Here wake instability alone establishes a street-like structure well downstream of the body, from which no vortices have been 'shed'.

A plot of $a_{2} / a_{1}$ (ratio of secondary-street scale to that of the primary street) versus Reynolds number, as shown for example by Matsui \& Okude (1980), has a great amount of scatter, particularly among different flow facilities. Furthermore, $a_{2} / a_{1}$ is exactly equal to two only for isolated cases. Hydrodynamic stability theory and its receptivity to facility-dominated disturbances adequately explains the scatter. As indicated by figure 12, the wake at a given location is unstable to a broad band of frequencies. If there exist relatively large background disturbances at specific frequencies within this band, these will most likely be the ones selected to amplify. It is then clear why such facility-dependency exists. Moreover, because a broad band of scales is amplified in the wake, and because the band of amplified frequencies shifts to lower values with increasing $x, a_{2}$ cannot be defined unambiguously; a plot of $a_{2} / a_{1}$ is thus of little use, perhaps even misleading.

How then to explain the observations of Matsui \& Okude (1981) whose smokevisualized pictures show examples of pairing of vortices on each side of the wake, e.g. starting at about 100 diameters downstream for $R e=140$ ? Based on our observations, we must conclude that in fact it is only the residual smoke patterns of 
the primary vortex street that are being amalgamated into the new instability and its resulting vortices.

It has been pointed out to us recently (Meiburg 1987) that the peaks in our velocity spectra are almost all multiples of some very low frequency. For example, in the $v^{\prime}$ velocity spectrum at $R e=150$ and $x / d=100$ (see figure 8 ), many of the peaks are exact multiples of a frequency $f_{\mathrm{k}} / 10$. At $R e=140$ (not shown here), where the effect is even more pronounced, Meiburg's figure 22 reveals that almost every spectral peak is an integral multiple of $f_{\mathrm{k}} / 23$. Meiburg performed inviscid calculations on an infinite array of vortices in a vortex-street pattern; namely, he applied a subharmonic perturbation to the two sides. He observed the appearance of a low frequency and its multiples, quite similar to our experimental spectra. Meiburg then argues that vortex pairing can occur with the subharmonic frequency playing a dominant role in the spectrum.

In fact, the initial vortices decay so rapidly that to model them by a periodic array is questionable. Meiburg's analysis is consistent with the general concept of the introduction of frequency into the spectrum by phase jitter. Such phase jitter may also be introduced by free-stream disturbances or three-dimensionality. These low frequencies would be selected for amplification by the changing instability window of the growing wake. Either scenario does not imply or require 'pairing' as the fundamental mechanism. The change in the character of the spectrum from $R e=150$ to $R e=500$ is striking. At $R e=500$ we have seen in figures 10 and 11 that the spectrum is broad and smooth, and shifts continuously to lower frequencies with increasing $\delta(x)$. (This type of spectrum occurs first at $R e=300$ (Roshko 1953).)

\subsection{Comparisons with the plane mixing layer}

A theme of this paper is that, as the wake develops, its large-scale structure (as measured by the stream wise spacing of vortices) increases in response to the stability characteristics of the thickening wake. It is of interest, therefore, to attempt comparison with the development of the plane mixing layer, for which the corresponding ideas have been discussed for some time (Ho \& Huerre 1984). Some authors emphasize the instability with respect to the mean profile, while others proceed from the stability of vortex arrays. These sometimes lead to controversial views of the processes.

In the case of the mixing layer it does appear that amalgamation processes are more vigorous than in the wake, that the developments proceed faster in some sense. This may be related to the different underlying growth laws: $\mathrm{d} \delta / \mathrm{d} x \approx \delta / x$ for the mixing layer but only $\frac{1}{2} \delta / x$ for the wake. In the slower-growing wake the growth decay cycles may be more prominent, more separated in scale, than in the mixing layer.

Possibly the most important differences between the mixing layer and the wake are that: $(a)$ in the former the mean (two-dimensional) vorticity is all of one sign, while in the latter the vorticity is of opposite signs on the two sides of the wake; $(b)$ the local characteristic Reynolds number of the wake remains essentially constant for the wake, while it increases continuously with downstream distance in the shear layer. The possibilities for vortex interactions, instabilities and structure are richer, more complex and have more time to develop in the wake. One result, apparently, is that the large organized structures are quasi-two-dimensional (spanwise organized) in the mixing layer but basically three-dimensional in the wake. This difference is illustrated in the experiment of Breidenthal (1980) who added a spanwise 
perturbation to a splitter plate to introduce three-dimensional disturbances into wakes and mixing layers. Three-dimensional, loop-like structure remained in the wake for large distances downstream; on the other hand the mixing layer reorganized itself into predominantly two-dimensional structure. This tendency toward threedimensionality in wakes complicates any possible amalgamation processes. Our observations under laminar conditions suggest a mechanism for developing these three-dimensionalities from a secondary parametric instability of the subharmonictype acting on the far-wake initially two-dimensional structures. Such mechansims may remain operative under turbulent wake conditions leading to the GrantTownsend vortex-pair eddies.

The authors are grateful for the financial support of the Office of Naval Research, US Navy, under Contract No. N00014-76-C-0260. We also thank Toshi Kubota for discussions of the wake stability problem and for generously allowing us to use his calculations of wake stability.

\section{REFERENCES}

Aref, H. \& Siggia, E. 1981 Evolution and breakdown of a vortex street in two dimensions. J. Fluid Mech. 109, 435-463.

Berger, E. \& Wille, R. 1972 Periodic flow phenomena. Ann. Rev. Fluid Mech. 4, 313-340.

Bevilaqua, P. 1975 Intermittency, the entrainment problem. ARL Tech. Rep. 75-0095, USAF.

Breidenthal, R. E. 1980 Response of plane shear layers and wakes to strong three-dimensional disturbances. Phys. Fluids 23, 1929-1934.

Cantwell, B. J. 1979 Coherent turbulent structures as critical points in unsteady flow. Arch. Mech. 31, 707-721.

Castro, I. 1971 Wake characteristics of two-dimensional perforated plates normal to an air-stream. J. Fluid Mech. 46, 599-609.

Crmbala, J. 1984 Large structure in the far wakes of two-dimensional bluff bodies. Ph.D. thesis, California Institute of Technology, Pasadena, California.

Cimbala, J., Nagib, H. \& Roshko, A. 1981 Wake instability leading to new large scale structures downstream of bluff bodies. Bull. Am. Phys. Soc. 26, 1256.

Champagne, F., Marasli, I. \& Wygnanski, I. 1982 A turbulent wake of a cylinder - some recent observations. Bull. Am. Phys. Soc. 27, 1163.

Corke, T., Koga, D., Drubka, R. \& Nagib, H. 1977 A new technique for introducing controlled sheets of streaklines in wind tunnels. IEEE Publication 77-CH 1251-8 AES.

Desruelle, D. 1983 Beyond the Kármán vortex street. M.S. thesis, Illinois Institute of Technology, Chicago, Illinois.

DURgin, W. \& KarLsson, S. 1971 On the phenomenon of vortex street breakdown. J. Fluid Mech. $48,507-527$.

Gaster, M. 1965 The role of spatially growing waves in the theory of hydrodynamic stability. Prog. Aero. Sci. 6, 251-270.

Gaster, M., Kit, E. \& Wygnanski, I. 1985 Large-scale structures in a forced turbulent mixing layer. J. Fluid Mech. 150, 23-39.

Gerrard, H. 1966 The three-dimensional structure of the wake of a circular cylinder. $J$. Fluid Mech. 25, 143-164.

Grant, H. L. 1958 The large eddies of turbulent motion. J. Fluid Mech. 4, 149-198.

Gupta, A., Laufer, J. \& Kaplan, R, 1971 Spatial structure in the viscous sublayer, J. Fluid Mech. 50, 493-512.

Нама, F. R. 1962 Streaklines in a perturbed shear flow. Phys. Fluids 5, 644-650.

Herbert, T. 1983 Secondary instability of plane channel flow to subharmonic three-dimensional disturbances. Phys. Fluids 26, 871-874. 
Herbert, T. 1984 Analysis of subharmonic route to transition in boundary layers. AIAA Paper 84-0009.

Ho, C.M. \& Hutrre, P. 1984 Perturbed free shear layers. Ann. Rev. Fluid Mech. 16, $365-424$.

НоOK ER, S. 1936 On the action of viscosity in increasing the spacing ratio of a vortex street. Proc. R. Soc. Lond. A 154, 67-89.

Hussain, A. K. M. F. \& Ramjee, V. 1976 Periodic wake behind a circular cylinder at low Reynolds numbers. Aero. Q. 27, 123-142.

KEFFER, J. 1985 The uniform distortion of a turbulent wake. J. Fluid Mech. 22, 135-159.

Lapple, C. E. 1961 The little things in life. Stanford Res. Inst. J. 5, 94-102.

Lessen, M. \& Singh, P. J. 1974 Stability of turbulent jets and wakes. Phys. Fluids 17, $1329-1330$.

MATSUI, T. \& OKUDE, M. 1980 Rearrangement of Kármán vortex street at low Reynolds numbers. $X V$ th International Congress of Theoretical and Applied Mechanics, University of Toronto, August, pp. 1-27.

Matsui, T. \& OKUDE, M. 1981 Vortex pairing in a Kármán vortex street. In Proc. Seventh Biennial Symposium on Turbulence, Rolla, Missouri.

MATSUI, T. \& OKUDE, M. 1983 Formation of the secondary vortex street in the wake of a circular cylinder. In Structure of Complex Turbulent Shear Flow, IUTAM Symposium, Marseille, 1982. Springer.

Mattingly, G. E. \& Criminale, W. O. 1972 The stability of an incompressible two-dimensional wake. J. Fluid Mech 51, 233-272.

MeIbURG, E. 1987 On the role of subharmonic perturbations in the far wake. J. Fluid Mech. 177, 83-107.

Morkovis, M. 1964 Flow around circular cylinders-a kaleidoscope of challenging fluid phenomena. In Proc. ASME Symposium on F'ully Separated Flows, Philadelphia, pp. 102-118.

Mumford, J. C. 1983 The structure of the large eddies in fully developed turbulent shear flows. Part 2. The plane wake. J. Fluid Mech. 137, 447-456.

Nagib, H. \& Desruelle, D. 1982 Controlled excitation of the far wake instability, Bull. Am. Phys. Soc. 27, 1193.

Nishioka, M. \& Sato, H. 1978 Mechanism of determination of the shedding frequency of vortices behind a cylinder at low Reynolds numbers. J. Fluid Mech. 89, 49-60.

Payne, F. \& Lumley, J. 1967 Large eddy structure of the turbulent wake behind a circular cylinder. Phys. Fluids Suppl. 10, S194-196.

Pierrenumbert, R. \& Widnall, S. 1982 The two- and three-dimensional instabilities of a spatially periodic shearlayer. $J$. Fluid Mech. 114, 59-82.

Robinson, A. C. \& Saffmax, P. G. 1982 Three-dimensional stability of vortex arrays. J. Fluid Mech 125, 411-427.

Roswko, A. 1953 On the development of turbulent wakes from vortex streets. NACA TN 2913 (see also, NACA Rep. 1191 (1959)).

Roshкo, A. 1976 Structure of turbulent shear flows: a new look. AIAA J. 14, 1349-1357.

Saffman, P. \& Schatzman, J. 1982 An inviscid model for the vortex-street wake. J. Fluid Mech. $122,467-486$.

Saric, W. \& Thomas, A. 1984 Experiments on the subharmonic route to turbulence in boundary layers. In Turbulence and Chaotic Phenomena in Fluids, (ed. T. Tatsumi), pp. 117-122. Elsevier.

Schatzman, J. 1981 A model for the von Kármán vortex street. Ph.D. thesis, California Institute of Technology, Pasadena, California.

TANedA, S. 1959 Downstream development of wakes behind cylinders. J. Phys. Soc. Japan 14, 843-848.

Townsend, A. A. 1956 The Structure of Turbulent Shear Flow. Cambridge University Press.

Towssend, A. A. 1966 The mechanism of entrainment in free turbulent flows. J. Fluid Mech. 26 , $689-715$.

Townsend, A. A. 1970. Entrainment and the structure of turbulent flow. J. Fluid Mech. 41, $13-46$. 
Townsend, A. A. 1979 Flow patterns of large eddies in a wake and in a boundary layer. J. Fluid Mech. 95, 515-537.

Tritton, D. J. 1959 Experiments on the flow past a circular cylinder at low Reynolds numbers. J. Fluid Mech. 6, 547-567.

Tritron, D.J. 1977 Physical Fluid Dynamics, p. 23. International Student Edition, Van Nostrand Reinhold.

Valensi, J. 1974 On the aerodynamic of porous sheets. In Omaggio a Carlo Ferrari.: Libreria Editrice Universitaria Levrotto \& Bella, Torino.

WELHS, D. 1973 On the existence of multiple Kármán vortex-street modes. J. Fluid Mech. 61, 199-205.

Winant, C. D. \& BRowand, F. K. 1974 Vortex pairing: the mechanism of turbulent mixing-layer growth at moderate Reynolds number. J. Fluid Mech. 63, 237-255.

Williamson, C. H. 1985 Evolution of a single wake behind a pair of bluff bodies. J. Fluid Mech. 159, 1-18.

Wlezien, R. 1981 The evolution of the low-wavenumber structure in a turbulent wake. Ph.D. thesis, Illinois Institute of Technology, Chicago, Illinois.

Wygnanski, I., Champagne, F. \& Marasli, B. 1986 On the large-scale structures in twodimensional small-deficit, turbulent wakes. $J$. Fluid Mech. 168, 31-71.

ZDravkovich, M. M. 1968 Smoke observations of the wake of a group of three cylinders at low Reynolds number. J. Fluid Mech. 32, 339-351.

Zdravkovich, M. M. 1969 Smoke observations of the formation of a Kármán vortex street. J. Fluid Mech. 37, 491-496. 